TID-4500, UC-4

Chemistry

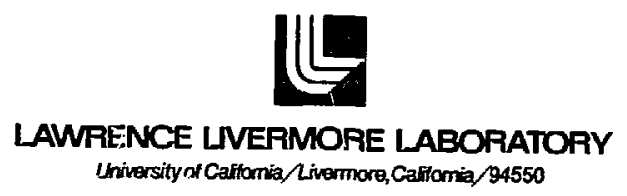

UCRL-51061, Vol. IV

\title{
COMPUTERIZED QUANTITATIVE ANALYSIS BY GAMMA-RAY SPECTROMETRY. VOL. IV. AUXILIARY PROGRAMS FOR GAMANAL
}

\author{
R. Gunnink \\ J. B. Niday
}

MS. date: June 1, 1972

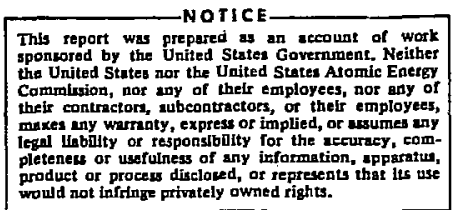




\section{Contents}

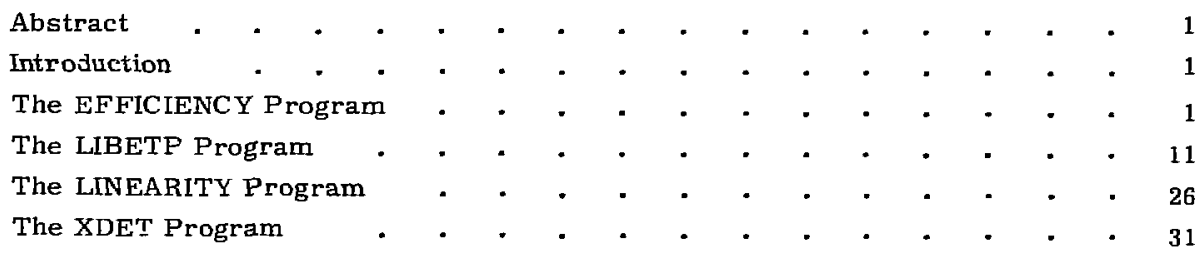




\section{Other Volumes}

VOL. I. Description of the GAMANAL Program (1972)

VOL. II. Source Listing of the GAMANAL Program (1971)

VOL. III. A User's Guide to GAMANAL (1971)

VOL. V. Decay-Scheme Data Library for GAMANAL (planned revision of UCrD-15439) 


\title{
COMPUTERIZED QUANTITATIVE ANALYSIS BY GAMMA-RAY SPECTROMETRY. VOL. IV. AUXILIARY PROGRAMS FOR GAMANAL
}

\begin{abstract}
This report describes and lists four small programs that are used in conjunction with the GAMANAL program. They are used to prepere a decay scheme library and to determine some of the parameters that characterize the counting systems.
\end{abstract}

\section{Introduction}

The GAMANAL program is primarily intended for complete computer analysis of high-resolution gamma-ray spectra obtained from mixtures of radioactive species such as fission products. For this purpose it examines the pulse-height data for "background" and "peak" regions, fits these peaks with the proper shape functions, and corrects for the effects of geometry, attenuation, and detector efficiency in evaluating the photon emission rate and for nonlinearities in the equipment in setting up an energy scale. These intermediate results are listed and plotted and, if no further data reduction is requested, the program goes on to the next spectrum. Otherwise, it proceeds to search a "library" of decay scheme information in order to make tentative assignments for each oî the peaks. This collection of "candidates" is examined for interactions between the photopeaks of the proposed nuclides and is divided into sets of species which interfere with each other at any point. A least-squares solution of the corresponding set of simultaneous equations is made and the amounts of various components originally present are calculated and listed, along with their estimated errors.

The following programs are used to prepare the decay scheme "library" and to determine the various parameters used to descrihe the linearity and the efficiency of a systen.

\section{The EFFICIENCY Program}

This auxiliary program fits a polynomial equation to a set of efficiency data for a Ge(Li) detector. The coefficients of the resulting polynomial can then be used to deBcribe the intrinsic efficiency of the detector.

Since we were unable to describe the efficiency adequately over the entire energy region with a single 6 th or 7 th order polynomial, we have chosen to use separate 
equations to describe the low energy region and the high energy reglon. A crossover energy, generally somewhere between 50 and $200 \mathrm{keV}$, is selected to give an optimum overall fit.

The input to this program consists of gamma-ray energies, the corresponding intrinsic efficiencies, and the associated errors, if greater than 1\%. Also specified are the terminal energy for the fitting range of the first polynomial and the starting energy for the fitting of the second polynomial. These specifications should result in an appreciable overlap within which a good crossover energy position can be chosen.

An equation of the following form will be fitted to the data (by the method of leastsquares):

$$
\text { In } \epsilon_{i}=\sum_{j=1}^{N} a_{j}\left(\ln E_{i}\right)^{j-1}
$$

where

$$
\begin{aligned}
& \varepsilon_{i}=\text { intringic efficiency at energy } E_{i} \\
& a_{j}=\text { polynomial coefficients }
\end{aligned}
$$

The resulting values of $a_{j}$ are placed on Type 5 cards as explained in Vol. III of this report. ${ }^{1}$

IJ. B. Niday and R. Gunnink, Computerized Quantitative Analysis by Gamma-Ray Spectroscopy. Vol. III, A User's Guide to GAMANAL, Lawrence Livermore Laboratory, Rept. UCAL-51061, Vol. III (1971). 


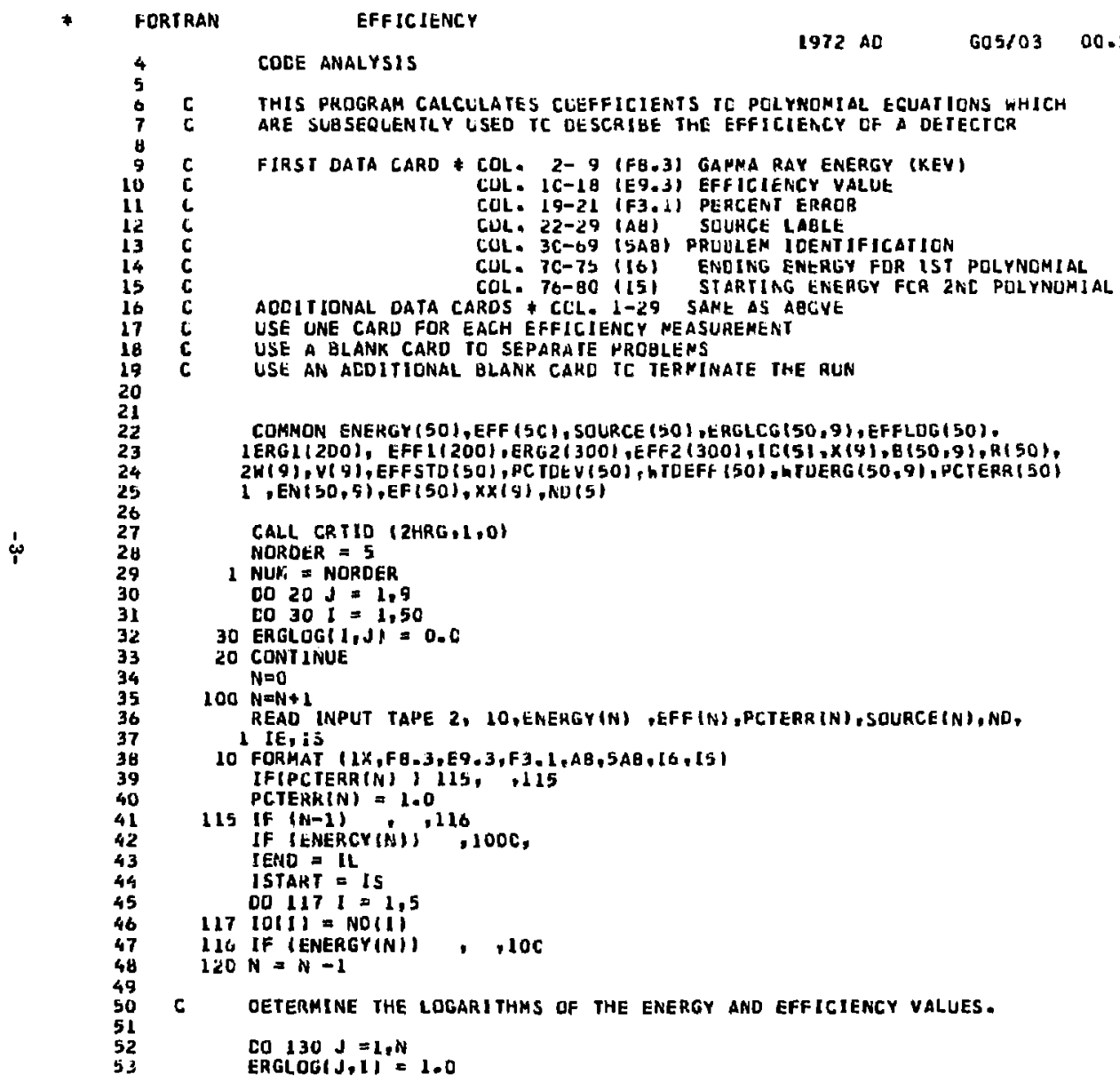

COCE ANALYSIS

C THIS PKOGRAM CALCULATES CLEFFICIENTS IC POLYROMIAL EGUATIONS WHICH ARE SUBSEQLENTLY USED TC DESCRIBE THE EFFICIENCY OF A DETECTCR

FIRST DATA CARD * COL. 2- 9 (FB.3) GAMMA RAY ENERGY (KEY)

CUL. IC-18 (E9.3) EFFICIENCY VALUE

CUL. 19-21 (F3.1) PERCENT ERROB

CUL. 22-29 (AB) SUUACE LABLE

COL. 3C-69 (SAB) PRUULEM IDENTIFICATION

CUL. 7C-73 (16) ENDING ENTRLY FDR IST POLYNCMIAL

COL. 76-80 (I5) STARTIAG ENERGY FCR 2NIC POLYNOMIAL

AOCITIONAL DATA CARDS + CCL. 1-29 SAME AS ABCYE

USE UNE CARD FOR EACH EFFICIENCY MEASUREAENT

USE A BLANK CARO TO SEPARATE MROGLENS

USE AN ACDITIONAL BLANK CAHD TC TERMINATE THE RUN

COAMON ENERGY (5O), EFF $(5 \mathrm{C})$, SOURCE $(50)$, ERGLC $(50,9)$, EFFLOG $(50)$,

1ERG (200), EFF1(200), ERG2(300), EFF $2(300), 10(5), \times(9), 8(50,9), R(50)$,

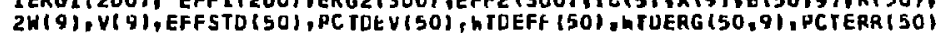

1 , EN $(50,5), E F(50), \times x(s), \mathrm{ND}(5)$

\section{CALL CRTID $(2 H R G, L, 0)$}

NORDER $=5$

1 NURY $=$ NORDER

$0020 \mathrm{~J}=1.9$

CO $30 \mathrm{I}=1,50$

30 ERGLOG $(1, \mathrm{~J})=0 . \mathrm{C}$

20 CONT INUE

$N=0$

$100 \mathrm{~N}=\mathrm{N}+1$

READ INPUT TAPE 2, 10,ENEKGY(N), EFF (N), PCTEPR (N), SOURCE(N), ND,

1 IE, is

10 FORMAT $(1 X, F B .3, E 9,3, F 3,1, A B, 5 A B,[6,[5)$

IF(PCTERR (N) IIS, 115

PCTERK(N) $=1.0$

115 If $(N-1), 116$

IF IENERCYINIS, $100 \mathrm{C}$,

IENID = IL

ISTART $=$ IS

DO $117=1,5$

$11710111=$ NO(II)

IIG IF (ENERGYINI), ,10C

$120 \mathrm{~N}=\mathrm{N}-1$

C OETERMINE THE LOGARITHMS OF the ENERGY AND EFfICIENCY VALUES.

CO $130 \mathrm{~J}=1, \mathrm{~N}$

ERGLOGIJ,II $=1.0$ 


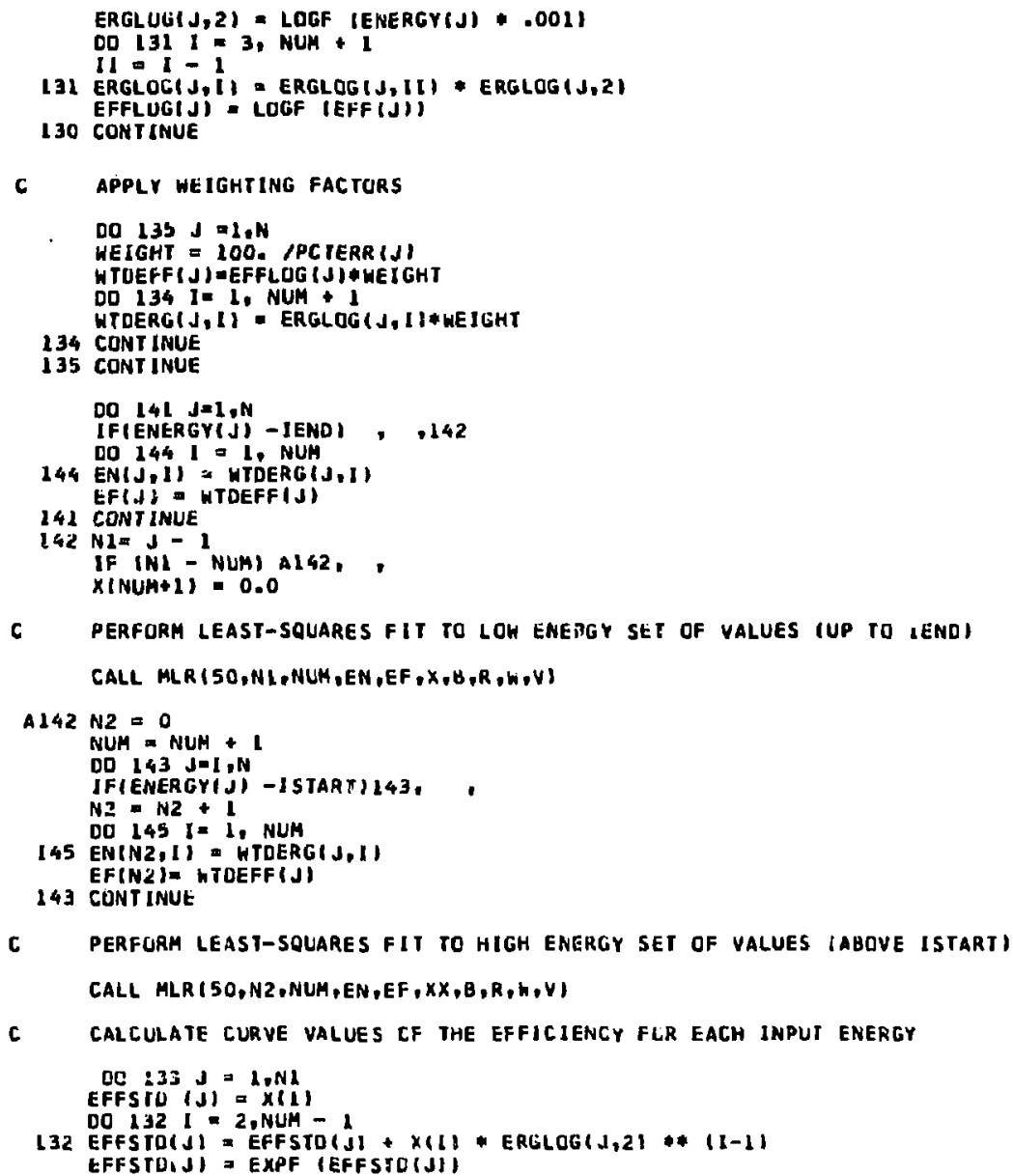




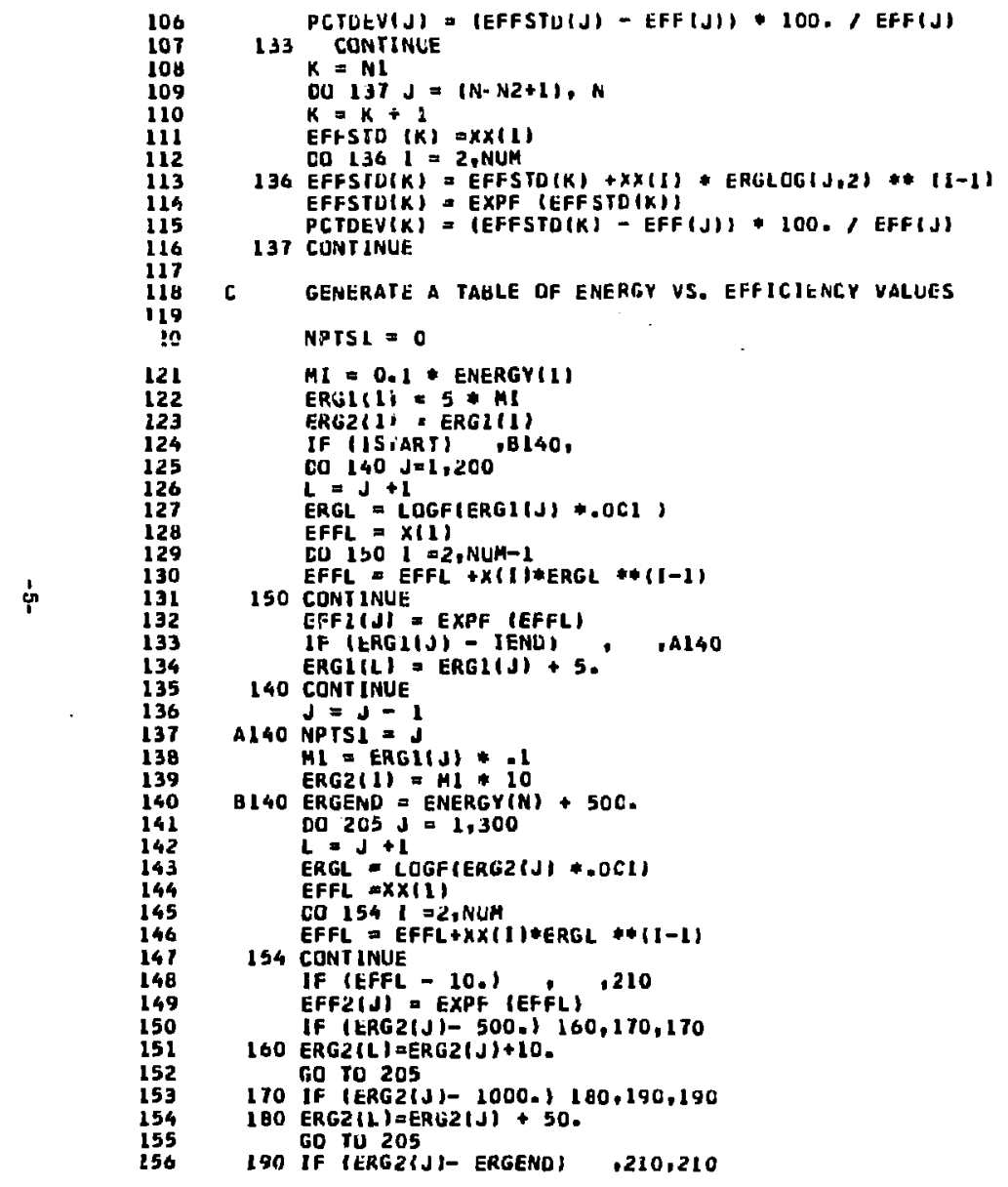


200 ERG2(L) = ERG2(d) + 200 .

205 CONTINUE

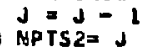

C DUTPUT DATA

HRITE DUTPUT YAPE 3,220, ID

220 FORMAT ILHI 19X,24HEFFICIENCY VS ENERGY OF $5 A 8$

HAITE OUTPUT TAPE 3,230 , NORDER

230 FORMATI//20X,2LHPOLYNOMIAL FIT IS OF, $11.8 H T H$ ORDER /

WRITE OUTPUT TAPE 3,240

240 FORMATI 2CX 5THENERGY EFFICIENCY PCTERR CALC EFF PCTOEY SOURCE

1 USEU /' WRITE DUTPU TAPE 3,250, (ENERLYII),EFF(I),PCTERR(1), EFFSTO(1), LPCTDEV(II, SDURCE (I), I I INI)

LPCTDEVTH

251 FDRMAT (I9X, 3OHCROSSCYER - PCLYMOMIAL IS OF $1 \mathrm{I}$, BHTH ORDEA $/$ ) NO $=N i+N 2-N$

WRITE JUTPUT TAPE 3,250 , (ENERGYI1), EFF (1), PCTERR(1), EFFSTU(1+NO)

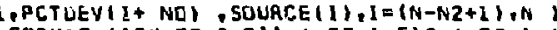

250 FDRMAT $(19 X, F 7.2, F 11.6, F 7,1, F 10.6, F 9.1,6 X, A B / 1)$ WRITE DUTPUT TAPE 3,255

255 FORMÄ̈I/20\%, 37HCOEFFICIEATS OF IHE PCLYMCMIALS ARE / 25x, $26 \mathrm{H}$ ILOW ENERGY HIGH ENERGY

WRITE OUTPUT TAPE $3,256,(\times(1), \times \times(1), I=1$, NUM $)$

26 FORMAT (20X, 2E 16.6$)$

nKITE DUTPUT TAPE 3,260

260 FORMAT 1LHL, L9X, 69HVALUES OF EFFICIEACY VS ENERGY CALUULATEC FROM If IT OF THE ABOVE DATA //

WRITE DUTPUT TAPE 3,265

265 FORMATI 22X,44HENERGY EFFICIENCY ENFTLY EFFICIENCY $A$ WRITE OUTPUT TAPE 3, 27C, (IERGL1I), EFF (11, 1 = 1,NPIS1), I (ERGL(1), EFF2(1), I = 1,NPTS2)

270 FORMATI $2 \mathrm{CX}$. FB.2, F14.7,F9.2, F14..7 11

CALL XSERTHIID,6,121

CALL AM INMX IEFF $2,1, N P T S 2,2, X M I N, X M A X$,

CALL GRIOC I4, 50,500C. $1,60 \mathrm{HLAERGY(HEVI}$

CALL XPLOTIERGL, I,NPTSI

$, 0, X Y I A,-5,1,10$ HEFFICIENCY , 21

CALL YPLOTIEFFI, I, 11

CALL XPLOTIERG2, L,NPTS2

CALL YPLOTIEFFR, $L, L$

CALL XPLDTIENERGY, $1, N$ )

CALL YPLOTIEEF, 1,01

GO TU 1

1000 CALL EXIT 
BANK

ADURESS BASE

0001157 MAIN.

0000005 MAIN.

TYPE

INTEGER

REAL

REAL II IERA LIIERAL LI IEP,AL STATEMENi LALEL
0001150 MAIN.

$0001151 \mathrm{MA} ! \mathrm{N}$.

** CLDE ANALYSIS *\#* SOURCE LINE REFERENCES

NAME
0
0.1
0.1
1

0000000

0000027 MAIN. 0001142 MAIN. 0001164 MAIN. 0001156 MAIN. 0001163 MAIN. OODOO6G MAIN. O000103 MAIN. 0000077 MAIN. 0001170 MA HN. 0000151 MAIN. 0000132 MAIN. 0000341 MAIN. 0000365 MAIN. 0000173 MAIN. 0000177 MAIN. 0000407 MAIN. D000434 MAIN. DO01171 MAIN. OOOOSIO MAIN. 0000226 MAIN. O000232 MAIN. 0000307 MAIN. 0000217 MAIN. 0000275 MAIN. 0000474 MAIN. OOO0S54 MAIN. DO00570 MALN. O000S75 MAIN. $0000601 \mathrm{MA}$ IN. 0000606 MAIN. 0001153 MAIN. 0000021 MAIN. 0000612 MAIN. DOOI LOL MAIN.

\begin{tabular}{|c|c|c|c|}
\hline $\begin{array}{l}\text { FUKMAT } \\
\text { STATEMENT } \\
\text { STATEMTNT } \\
\text { REAL } \\
\text { REAL } \\
\text { RLAL } \\
\text { STATEMENT } \\
\text { STATEMENT } \\
\text { STATEMENT } \\
\text { INTEGER } \\
\text { STATEMENT } \\
\text { STATEMENT } \\
\text { STATEMENT } \\
\text { STATEMENT } \\
\text { STATEMENT } \\
\text { STATEMENT } \\
\text { STATEMENT } \\
\text { STATEMENT } \\
\text { STATEMENT } \\
\text { INTEGER } \\
\text { STATEMENT } \\
\text { STATEMENT } \\
\text { STATEMENT } \\
\text { STATEMEIYT } \\
\text { STATEMENT } \\
\text { STATEMENT } \\
\text { STATEMENT } \\
\text { STATEMENT } \\
\text { STATEMENT } \\
\text { STATEMENT } \\
\text { STATEMENT } \\
\text { STATEMENT } \\
\text { REAL } \\
\text { INTEGER } \\
\text { STATEMENT } \\
\text { STATEMENT } \\
\text { AEAL } \\
\text { STATEMENT }\end{array}$ & 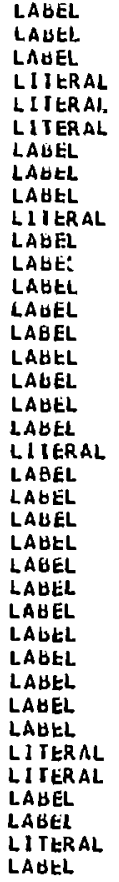 & $\begin{array}{l}1 \\
1 \\
1 \\
1\end{array}$ & $\begin{array}{l}10 \\
100 \\
1000 \\
1000 \\
100 . \\
10 . \\
115 \\
116 \\
117 \\
12 \\
120 \\
130 \\
131 \\
132 \\
133 \\
134 \\
135 \\
136 \\
137 \\
14 \\
140 \\
141 \\
142 \\
143 \\
144 \\
145 \\
150 \\
154 \\
160 \\
170 \\
180 \\
190 \\
1.0 \\
2 \\
20 \\
200 \\
200 . \\
205\end{array}$ \\
\hline
\end{tabular}

$152 \quad 155 \quad 158 *$
I NAME IN NO EXECUTABLE INSTRUCTION C GENOIES ORIGINAL COUNT IN CLICHE * uendies name defined ox lagel use 201

$\begin{array}{rrrrrrrrrr}27 & 34 & 85 & 120 & 145 & 201 & & & & \\ 32 & 79 & & & & & & & & \\ 121 & & & & & & & & \\ 27 & 29 * & 30 & 31 & 35 & 41 & 45 & 48 & 52 & 53 \\ 55 & 36 & 63 & 66 & 66 & 71 & 73 & 11 & 79 & 166 \\ 87 & 89 & 90 & 101 & 102 & 103 & 104 & 109 & 110 & 111 \\ 113 & 121 & 122 & 123 & 123 & 125 & 126 & 128 & 129 & 130 \\ 136 & 39 & 141 & 142 & 144 & 146 & 159 & 172 & 177 & 182 \\ 190 & 190 & 193 & 193 & 195 & 195 & 190 & 197 & 197 & 198 \\ 199 & 199 & 200 & 201 & 202 & & & & & \end{array}$

$106 \quad 115$

39 41*

47*

59*

$57 *$

$104 *$

$107 *$

684

69*

$113 *$

$116 *$

$135 *$

$135 *$
$76 *$

77*

74*

914

$131 *$

147*

$151 *$

$150 \quad 1530$

$154 *$

153

53
54

$33 *$

25

$57 \quad 103$

104

112

113

129

145

195

$158 \%$ 



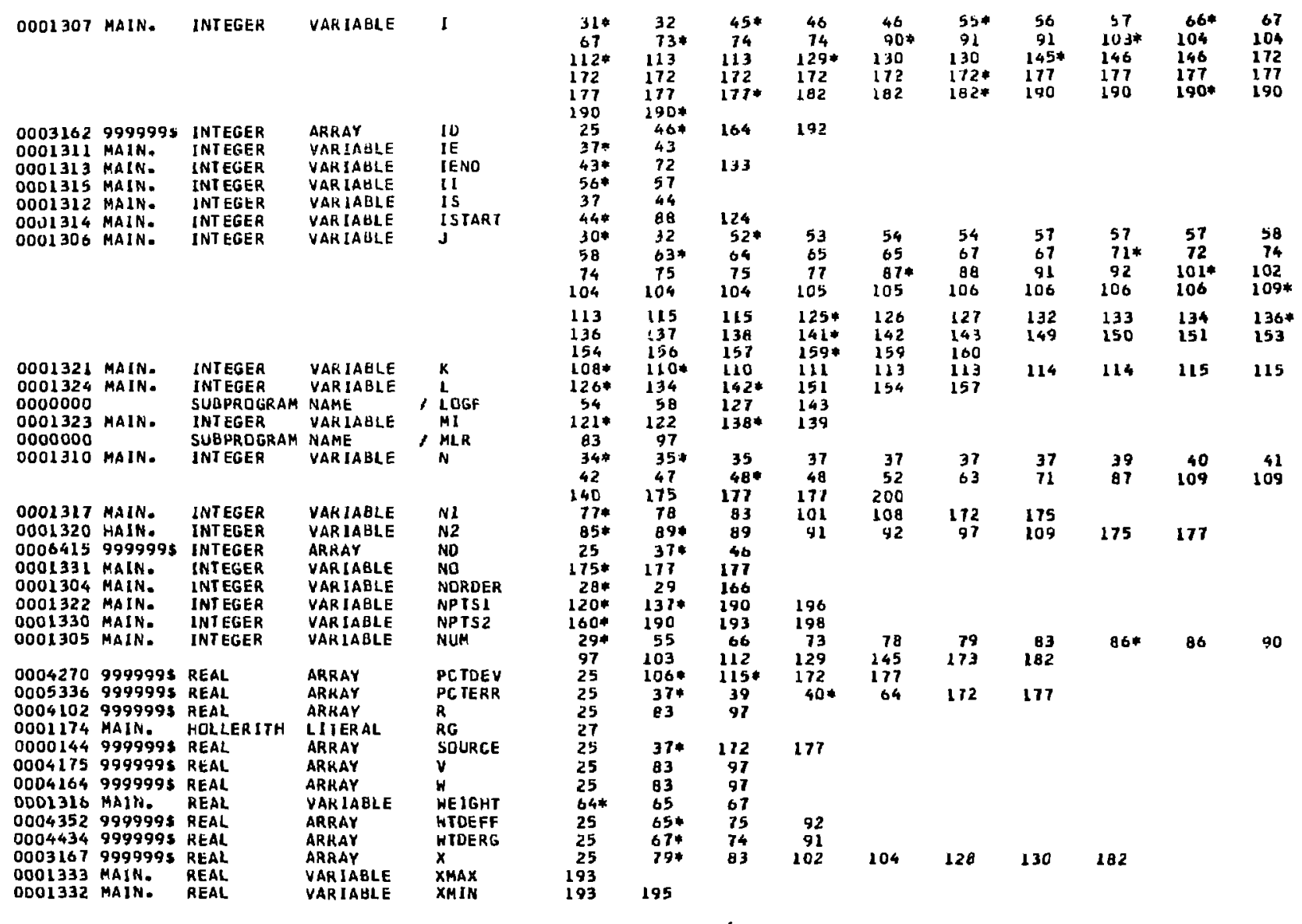




\begin{tabular}{|c|c|c|c|c|c|c|c|c|c|c|c|}
\hline $\begin{array}{l}0000000 \\
0000000 \\
0006404 \\
0000000 \\
0001154 \\
0001161 \\
0001173\end{array}$ & $\begin{array}{l}9999995 \\
\text { MAIN. } \\
\text { MAIN. } \\
\text { MAIN. }\end{array}$ & $\begin{array}{l}\text { SUHPRQGRAM } \\
\text { SUHPROGRAM } \\
\text { REAL } \\
\text { SUYPROGRAM } \\
\text { REAL } \\
\text { REAL } \\
\text { REAL. }\end{array}$ & $\begin{array}{l}\text { NAME } \\
\text { NAME } \\
\text { ARMAY } \\
\text { NAME } \\
\text { LI TERAL } \\
\text { LITERAL } \\
\text { L I TERAL }\end{array}$ & $\begin{array}{l}1 \\
1\end{array}$ & $\begin{array}{l}\text { XPLOT } \\
\text { XSERTH } \\
\text { XX } \\
\text { YPLOT } \\
.001 \\
.1 \\
.5\end{array}$ & $\begin{array}{r}196 \\
192 \\
25 \\
197 \\
54 \\
138 \\
195\end{array}$ & $\begin{array}{r}198 \\
97 \\
199 \\
127\end{array}$ & $\begin{array}{l}200 \\
111 \\
201 \\
143\end{array}$ & 113 & 144 & 146 \\
\hline
\end{tabular}




\section{The LIBETP Program}

In order to identify and evaluate quantitatively the nuclides represented by a spectrum, one must have access to a library compatible with the method used in reducing the spectral data. This program generates a decay-scheme library that is suitable for GAMANAL and then records it on magnetic tape.

Two blocks of data are read in from cards. The first is on a set of cards identified by a 1 in column 1 . There is a card for each of the nuclides which contains its nuclide number (combined atomic number and mass number), its half-life, and the nuclide numbers of any parent or daughter nuclides that should be considered with it.

The second set consists of one card for each gamma ray and has a 2 in column 1. These cards carry the nuclide number of the source, the energy and branching intensity of the gamma ray, an еrгor estimate for this branching intensity if greater than $1 \%$, and a list of up to four other gamma rays that should be found in association with this one. If these "associative" gamma rays are in the decay scheme of the parent or the daughter nuclide, they are so tagged.

LIBETP rearranges and cross-references this input information in such a way that GAMANAL will have no need to search for correlated information. In this process the gamma-ray list is first ordered according to increasing energy. An associated array is created for the branching intensity of each gamma ray and another for a packed set of indices to the locations of the corresponding associative gamma rays and of the source nuclide. The list of nuclides is also structured for easy reference to the corresponding half-lives and any parent-daughter relationship.

To reckon with two irregularities that are commonly encountered, the following conventions are used: 1) the mass number of meta-stable nuclides is increased by 300 and 2) the branching intensities associated with double and single-escape, pair-peaks are tagged respectively as $10^{-10}$ and $10^{-20}$ times the full-energy, peak-branching intensities.

After the data have been properly organized and packed for economy of space, the arrays are written onto magnetic tape in a suitable format for use by GAMANAL. 


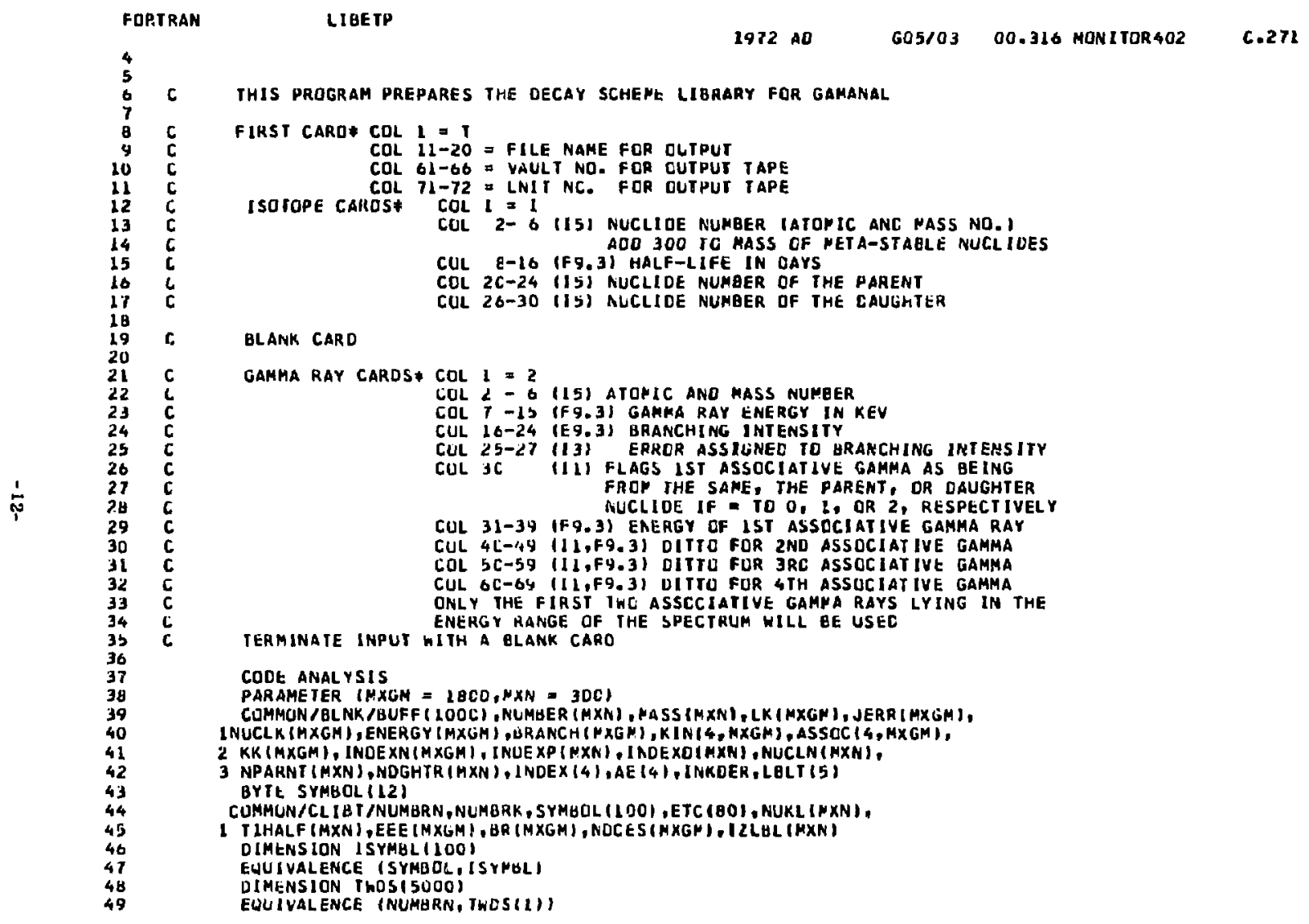


C FOLLOWING ARE THE ELEMENT SYHUOLS

DATA I ISYMUL $=200 \mathrm{OH}$ HHEL IUE O C N C FAENAMGALSI P SCLAR KCASCTI YCR IMNFECON ICUZNGALEAS SEBRKRRB 5R YZRAHMCTCRLRHPCAGCDIASNSUTE IXECSBALA 2CEPRMUPMSMEUGDTBDYHOERT YYULUHF TA WRECSIRPTAUHG TLPBBIPCATANFHRAACJH 3PA UNPPUAMCMBKCFESFM.

$$
\begin{aligned}
& \text { CALL OONLGE (BUFF, 1000,2, INKDER) } \\
& \text { IF I INKCER-II) }
\end{aligned}
$$

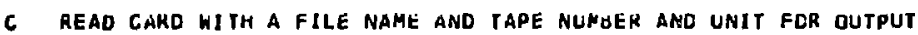

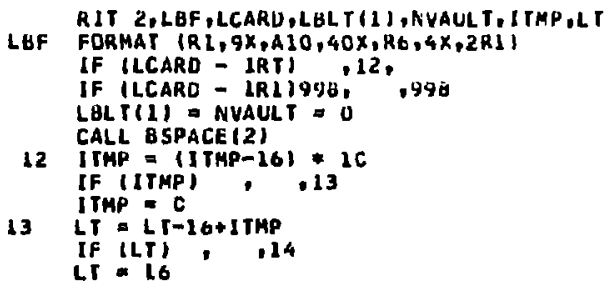

C READ hALF-LIFE AND PARENT-DAUGHTER INFCRMATION FOR THE YARIOUS NUCLIDES IINDEX THESE ARRAYS BY $\mathrm{N}$ \}

$14 N=0$

READ INPUT TAPE $2,30,10$, NUCLN(M), TIHALF (N), NPARHT (N), NEGHTR(N)

30 FORHAT $(11,15,2 x, E 9,3,3 x, 15,(x, 15)$

DECOUE 35, (NUCLN(N)), 36, (NUMBER (N), MASS (NI)

35 FORMAT (I5)

36 FURMAT (12, (3)

IF IID $-1,40,20,40$

40 NUMBKN $=N-1$

C READ ENERGY AND BRANCHING DATA FCR a GAMMA RAY AND ENERGIES OF UP TO FOUR ASSUCIATIVE GAHMA RAYS (INDEX ARRAYS BY $K$ )

$50 K=0$

$0 K=K+1$

READ INPUT TAPE 2,60, 10 , NUCLK(K), ENERGY(K),GRANCH(K), JERR(K),

1 IKIN(J,K),ASSOC $(J, K), J=1,4)$

60 FDRHAT III,I $, 2 E 9.3,13,2 x, 4111, F 9,31)$

If $110-2 ; 70,50,70$

70 NURBRK $=K-I$ 


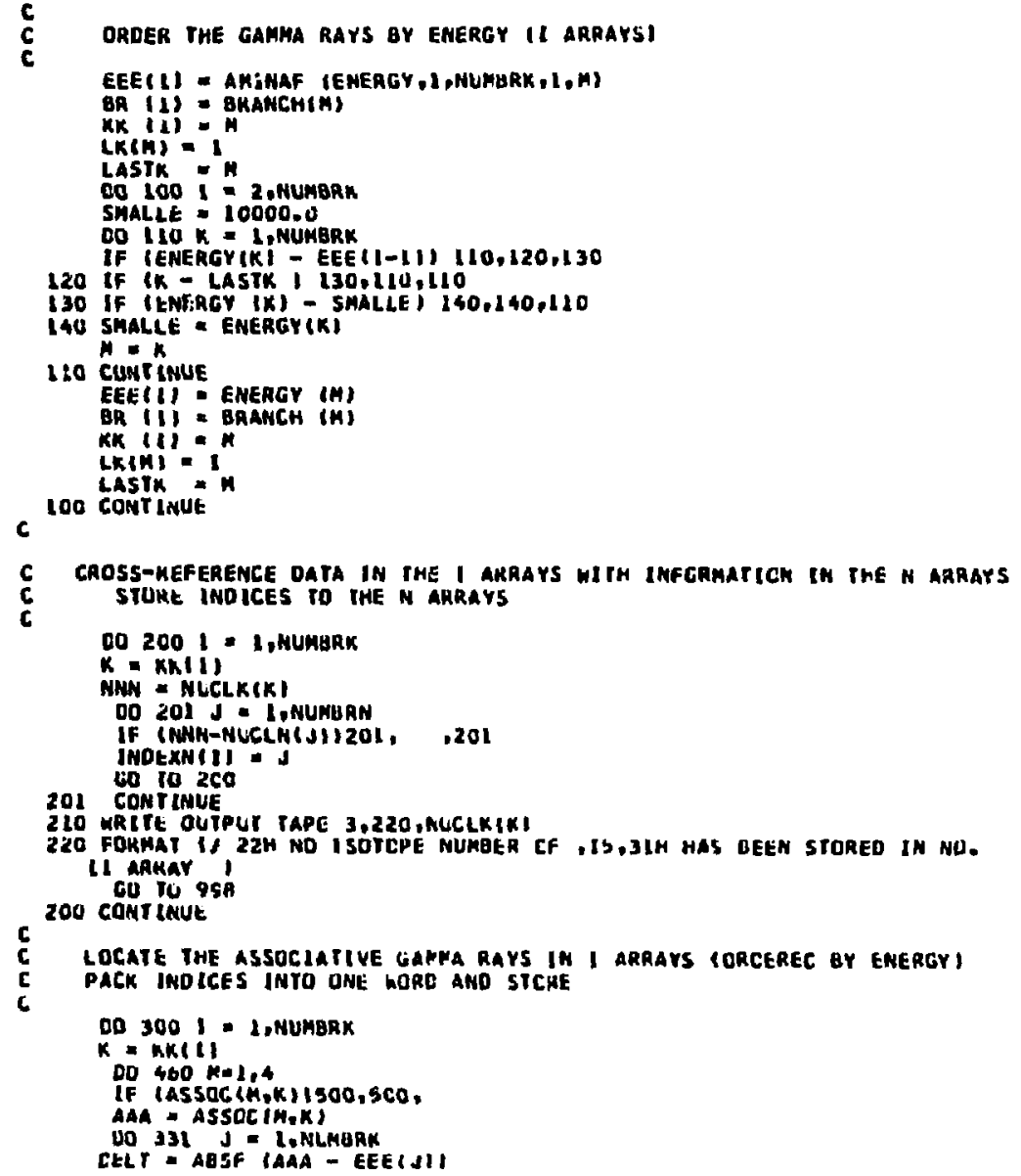

C CAOSS-MEFERENCE DATA IN THE I MHRAYS WITH IMFGRMATICK IN THE $N$ MARAYS

C STORE INDICES TO THE M ARRAYS

c 002001 1.hUnaRK

\section{$K=\operatorname{RK}(1)$}

DO $201 \mathrm{~J}$ IN NUABR

IF INHA-NUSCHCJII2OL, 201

JMOEXN(I) $=J$

201 ConTIuUE

210 WRITE OUIPUR TAPE 3.220 MUCLKIK

220 FOKMAT If $22 \mathrm{H}$ NO ISOTCPE MUREER CF, Ib.3IM HAS BEEN STOREO IN NU. 11 ankar ! go Tu 998

C LOCATE THE ASSOClative garra RAYS IH ARRAYS TORCEREC GY ENERGYI PACK INDICES INTO ONE hORE AMD STCHE 


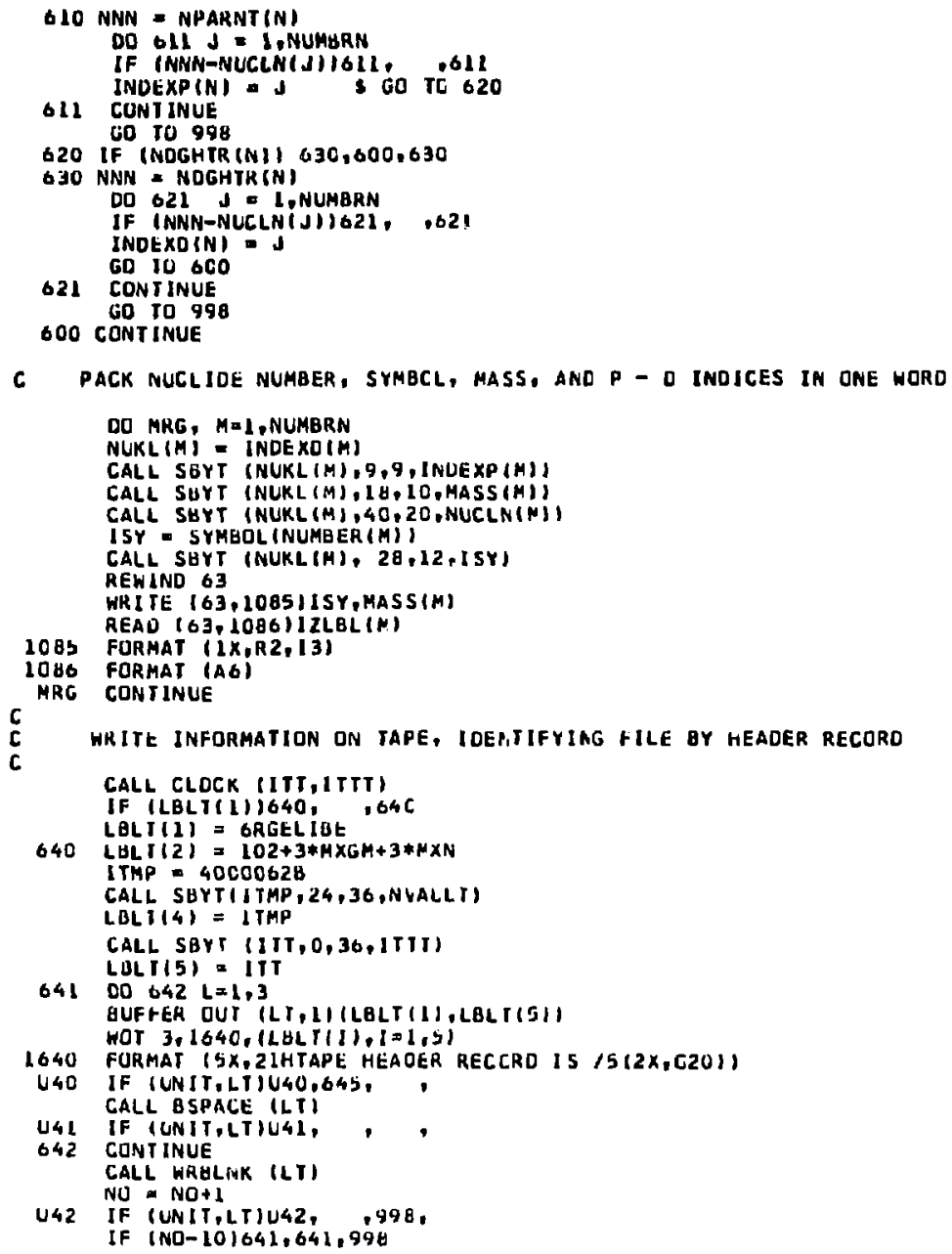




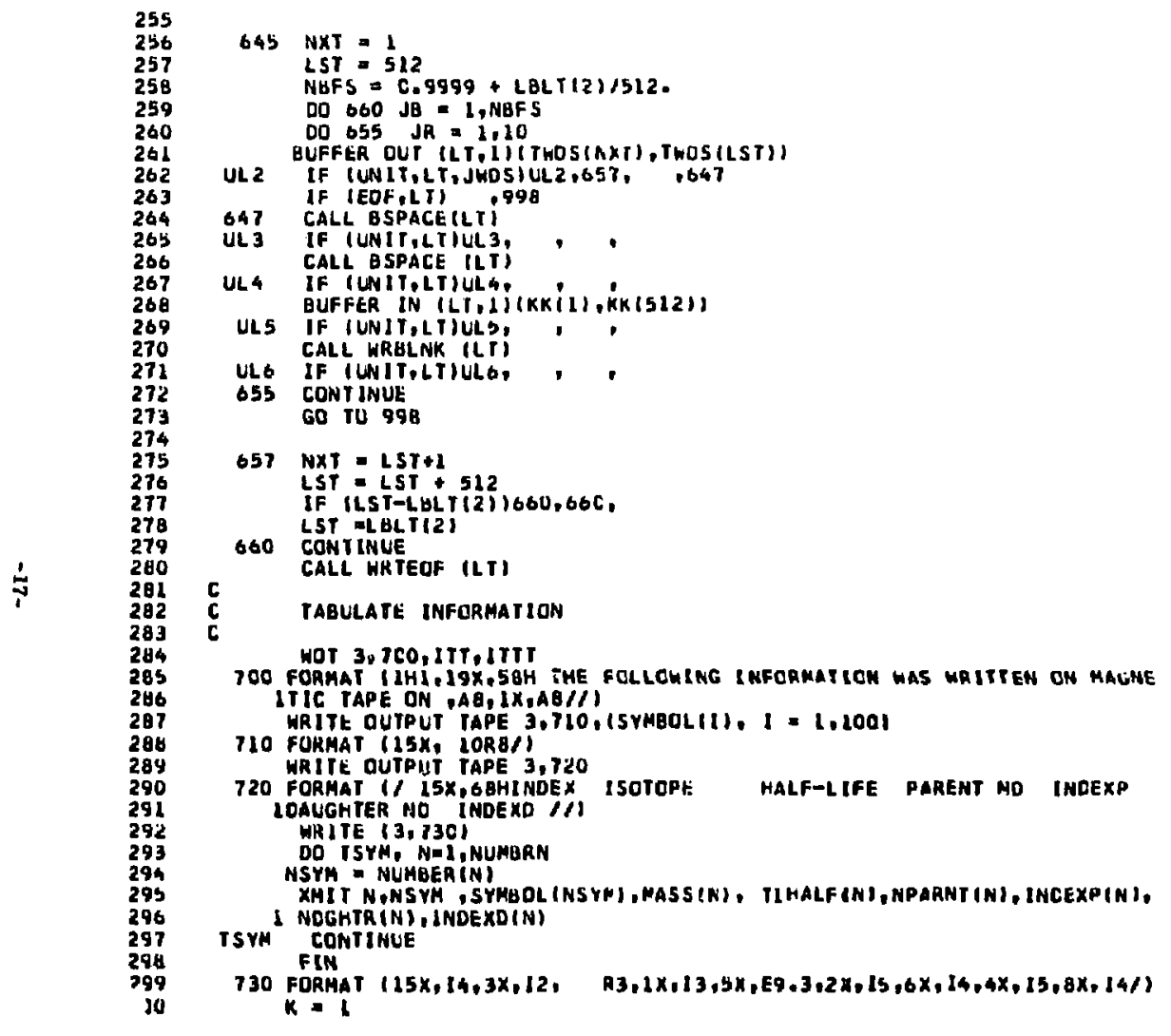




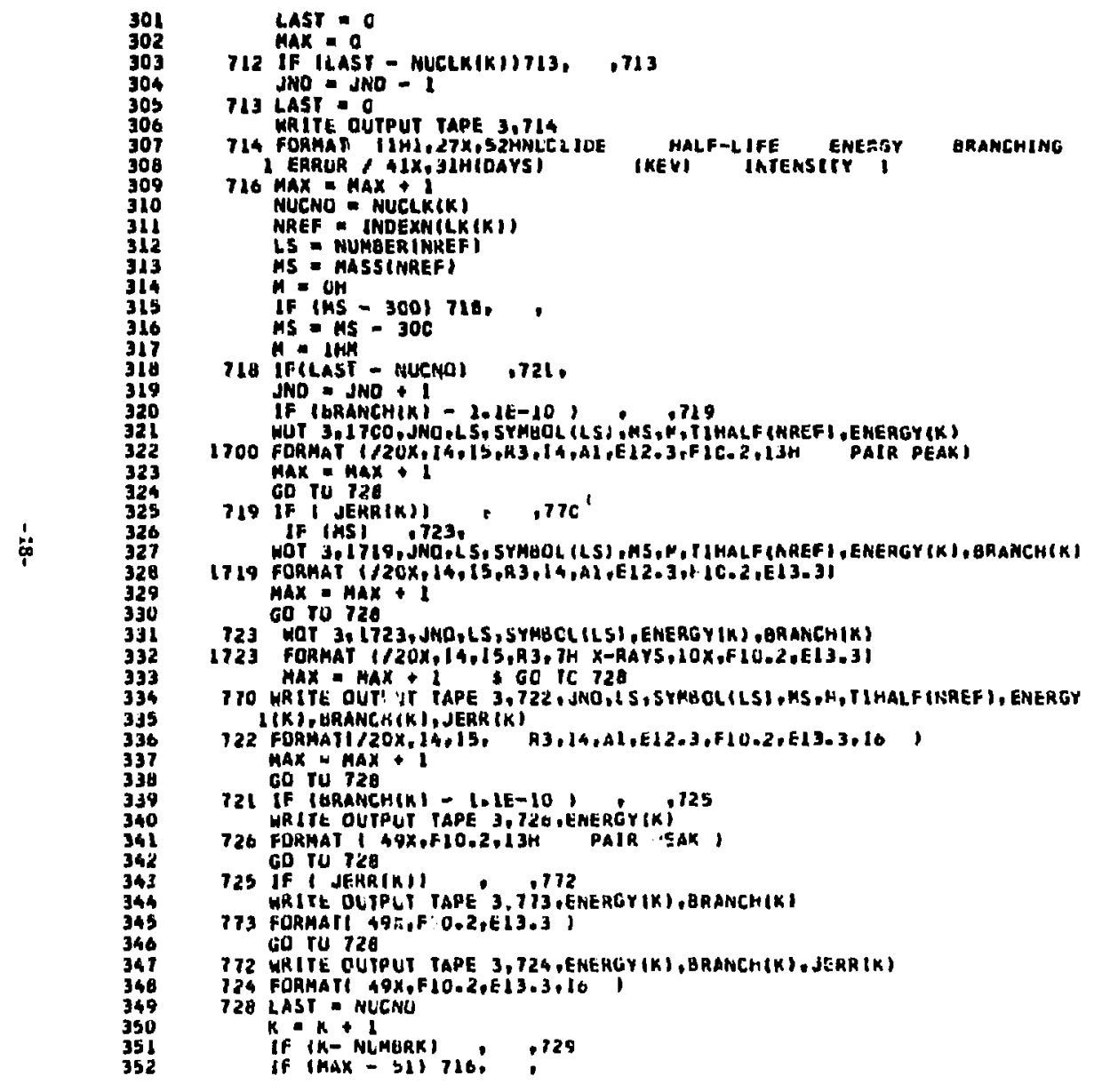




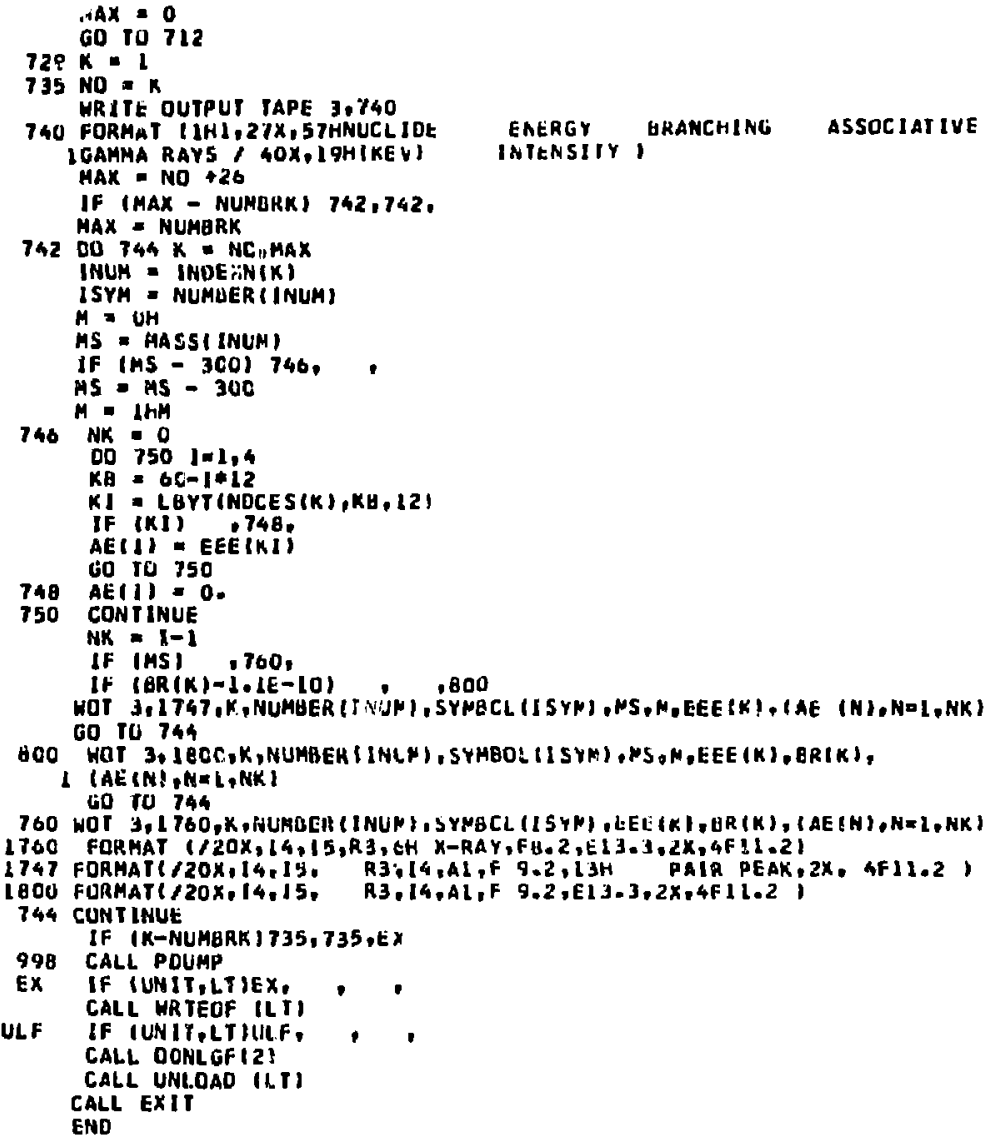





\begin{tabular}{|c|c|c|c|c|c|c|c|c|c|c|c|c|c|}
\hline $\begin{array}{l}0000000 \\
0000561 \\
0000352\end{array}$ & $\begin{array}{l}\text { MAIN. } \\
\text { MAII }\end{array}$ & $\begin{array}{l}\text { FDRMAT } \\
\text { STATEMENT } \\
\text { STATEMENT }\end{array}$ & $\begin{array}{l}\text { LABEL } \\
\text { LABEL } \\
\text { LABEL }\end{array}$ & $\begin{array}{ll}30 \\
/ & 300 \\
331\end{array}$ & $\begin{array}{r}224 \\
132 \\
287 \\
347 \\
79 \\
38 \\
146\end{array}$ & $\begin{array}{c}160 \\
289 \\
357 \\
80 \\
141 \\
148\end{array}$ & $\begin{array}{l}178 \\
292 \\
383 \\
194{ }^{\circ} \\
151 *\end{array}$ & $\begin{array}{l}183 \\
306 \\
386 \\
315\end{array}$ & $\begin{array}{l}191 \\
321 \\
388 \\
316\end{array}$ & $\begin{array}{l}237 \\
327\end{array}$ & 369 & $\begin{array}{l}243 \\
335\end{array}$ & $\begin{array}{l}245 \\
340\end{array}$ \\
\hline $\begin{array}{l}0000356 \\
0000000 \\
0000432 \\
0000000 \\
0000374 \\
0000373 \\
0000401 \\
0000408 \\
0000000 \\
0000122 \\
0000513 \\
0002043 \\
0000000 \\
0000447 \\
0000454 \\
0000461 \\
0000466 \\
0000534 \\
0000000 \\
0000000 \\
0000467 \\
0000000 \\
0000000 \\
0000125 \\
0000532 \\
0000000 \\
0000000 \\
0000000 \\
0000000 \\
0000000 \\
0000637 \\
0000573 \\
0000607 \\
0000614 \\
0000632 \\
0002042 \\
000061.6 \\
0000736 \\
0000751 \\
0001000 \\
0001021 \\
0001054 \\
01901104 \\
0001111\end{array}$ & $\begin{array}{l}\text { MAIN. } \\
\text { MAIN. } \\
\text { MAIN. } \\
\text { MAIN. } \\
\text { MAIN. } \\
\text { MAIN. } \\
\text { MAIN. } \\
\text { MAIN. } \\
\text { MAIN. } \\
\text { MAIN. } \\
\text { MAIN. } \\
\text { MAIN. } \\
\text { MAIN. } \\
\text { MAIN. } \\
\text { MAIN. } \\
\text { MAIN. } \\
\text { MAIN. } \\
\text { MAIN. } \\
\text { MAIN. } \\
\text { MAIN. } \\
\text { MAIN. } \\
\text { MAIN. } \\
\text { MAIN: } \\
\text { MAIN. } \\
\text { MAIN. } \\
\text { MAIN. }\end{array}$ & $\begin{array}{l}\text { STATEMENT } \\
\text { FORMAT } \\
\text { STATEMENT } \\
\text { FDRMAT } \\
\text { STATEMENT } \\
\text { STATEMENT } \\
\text { STATEMENT } \\
\text { STATEMENT } \\
\text { INTEGER } \\
\text { STATEMENT } \\
\text { STATEMENT } \\
\text { BOOLEAN } \\
\text { FORMAT } \\
\text { STATEMENT } \\
\text { STATEMENT } \\
\text { STATEMENT } \\
\text { STATEMENT } \\
\text { STATEMENT } \\
\text { FORHAT } \\
\text { INTEGER } \\
\text { STATEMENT } \\
\text { FORHAT } \\
\text { INTEGER } \\
\text { STATEMENT } \\
\text { STATEMENT } \\
\text { INTEGER } \\
\text { INTEGER } \\
\text { INTEGER } \\
\text { REAL } \\
\text { FORHAT } \\
\text { STATEMENT } \\
\text { STATEMENT } \\
\text { STATEMENT } \\
\text { STATEMENT } \\
\text { STATEMENT } \\
\text { INTEGER } \\
\text { STATEMENT } \\
\text { STATEMENT } \\
\text { STATEMENT } \\
\text { STATEMENT } \\
\text { STATEMENY } \\
\text { STATEMENT } \\
\text { STATEMENT } \\
\text { STATEMENT }\end{array}$ & $\begin{array}{l}\text { LABEL } \\
\text { LABEL } \\
\text { LABEL } \\
\text { LABEL } \\
\text { LABEL } \\
\text { LABEL } \\
\text { LABEL } \\
\text { LABEL } \\
\text { LITERAL } \\
\text { LABEL } \\
\text { LABEL } \\
\text { LITERAL } \\
\text { LABEL } \\
\text { LABEL } \\
\text { LABEL } \\
\text { LABEL } \\
\text { LABEL } \\
\text { LABEL } \\
\text { LABEL } \\
\text { LITERAL } \\
\text { LABEL } \\
\text { LABEL } \\
\text { LITERAL } \\
\text { LABEL } \\
\text { LABEL } \\
\text { LITERAL } \\
\text { LITERAL } \\
\text { LITERAL } \\
\text { LITERAL } \\
\text { LABEL } \\
\text { LABEL } \\
\text { LABEL } \\
\text { LABEL } \\
\text { LABEL } \\
\text { LABEL } \\
\text { LITERAL } \\
\text { LABEL } \\
\text { LABEL } \\
\text { LABEL } \\
\text { LABEL } \\
\text { LADEL } \\
\text { LABEL } \\
\text { LABEL } \\
\text { LABEL }\end{array}$ & 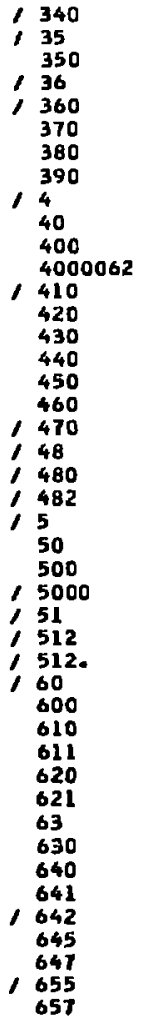 & $\begin{array}{c}152 * \\
81 \\
150 \\
81 \\
153 \\
155 \\
155 \\
159 \\
42 \\
84 \\
159 \\
238 \\
160 \\
172 \\
172 \\
172 \\
173 \\
143 \\
178 \\
193 \\
170 \\
183 \\
42 \\
91 * \\
144 \\
48 \\
352 \\
257 \\
258 \\
93 \\
198 \\
199 \\
201 \\
199 \\
208 \\
225 \\
206 \\
235 \\
243 * \\
243 \\
247 \\
262 \\
260 \\
262\end{array}$ & $\begin{array}{c}82 * \\
170 * \\
83 * \\
157 * \\
155 \\
158 * \\
159 \\
42 \\
84 \\
183 * \\
164 * \\
173 * \\
174 * \\
175 * \\
173 \\
173 \\
181 * \\
177 * \\
186 * \\
242 \\
95 \\
144 \\
\\
268 \\
\\
94 * \\
206 \\
199 \\
202 \\
203 \\
209 \\
226 \\
206 \\
235 \\
254 \\
250 * \\
256 * \\
264 * \\
272 * \\
275 *\end{array}$ & $\begin{array}{l}244 \\
182 \\
276 \\
373 \\
211 \\
200 * \\
202 \\
206 \\
209 \\
227 \\
207 * \\
237 \% \\
254\end{array}$ & $\begin{array}{l}174 \\
175 \\
245 \\
187 * \\
214 * \\
204 * \\
212 *\end{array}$ & $\begin{array}{l}241 \\
93\end{array}$ & 更 & $\begin{array}{l}190 \\
176 *\end{array}$ & 240 & 372 \\
\hline
\end{tabular}




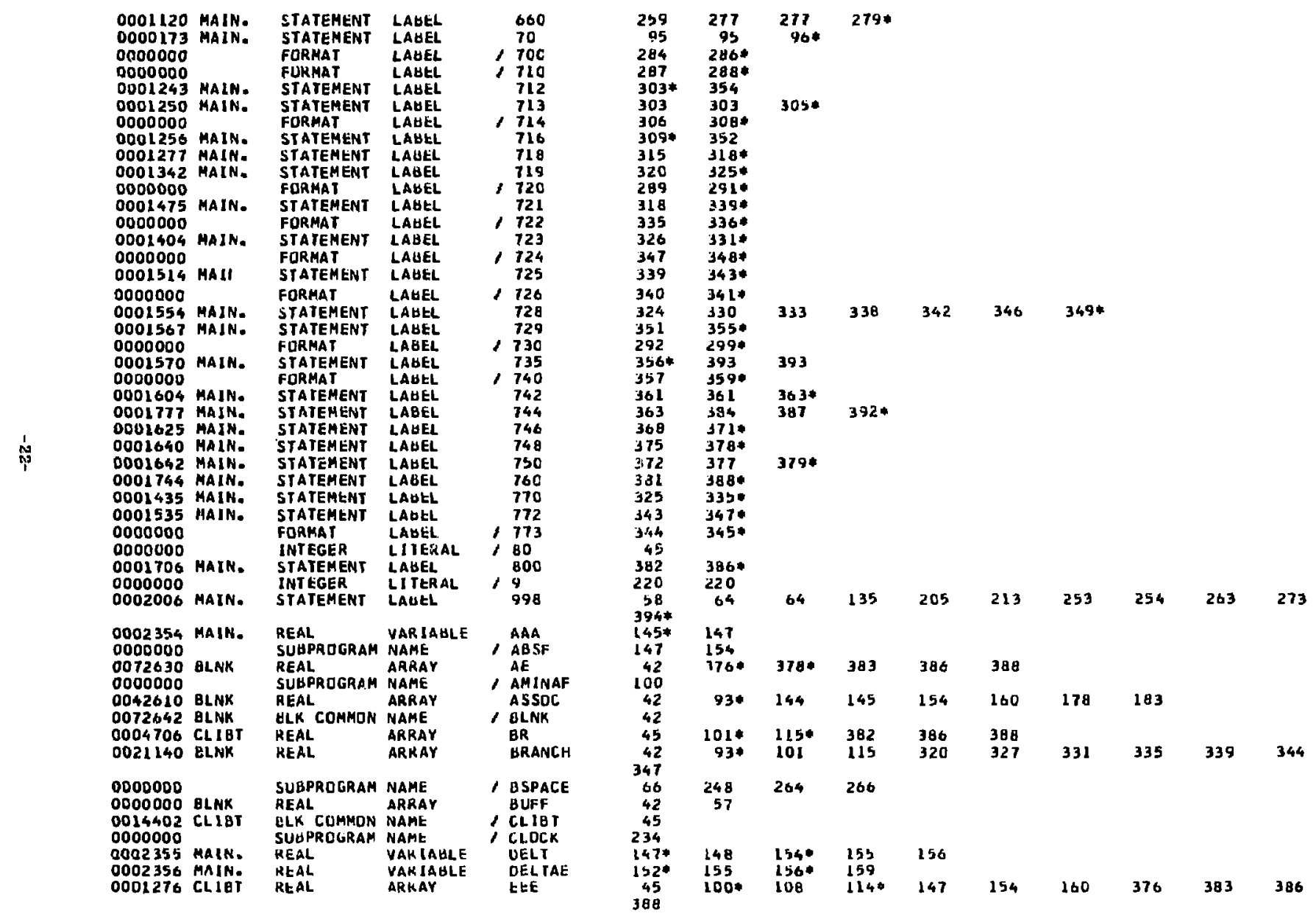




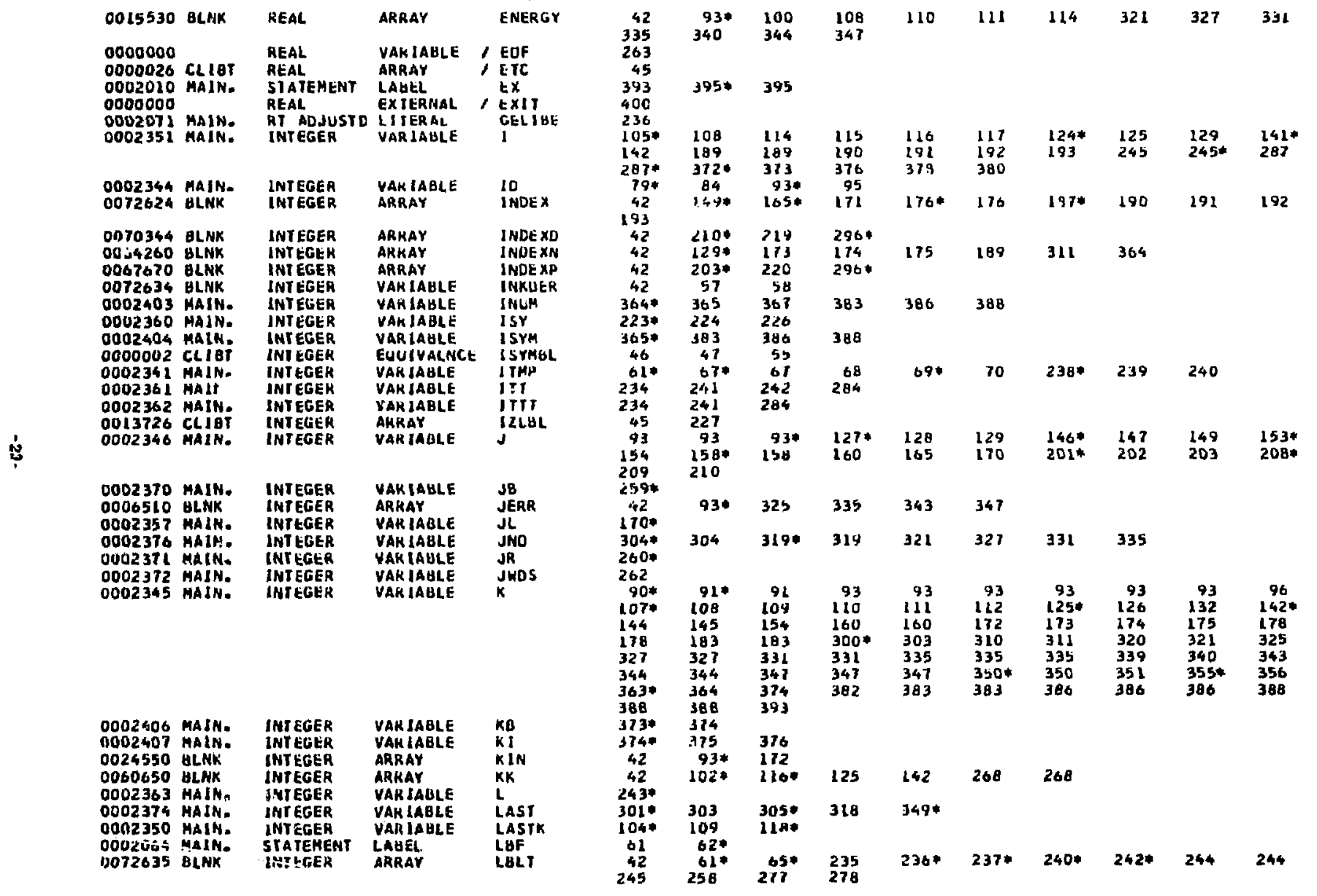




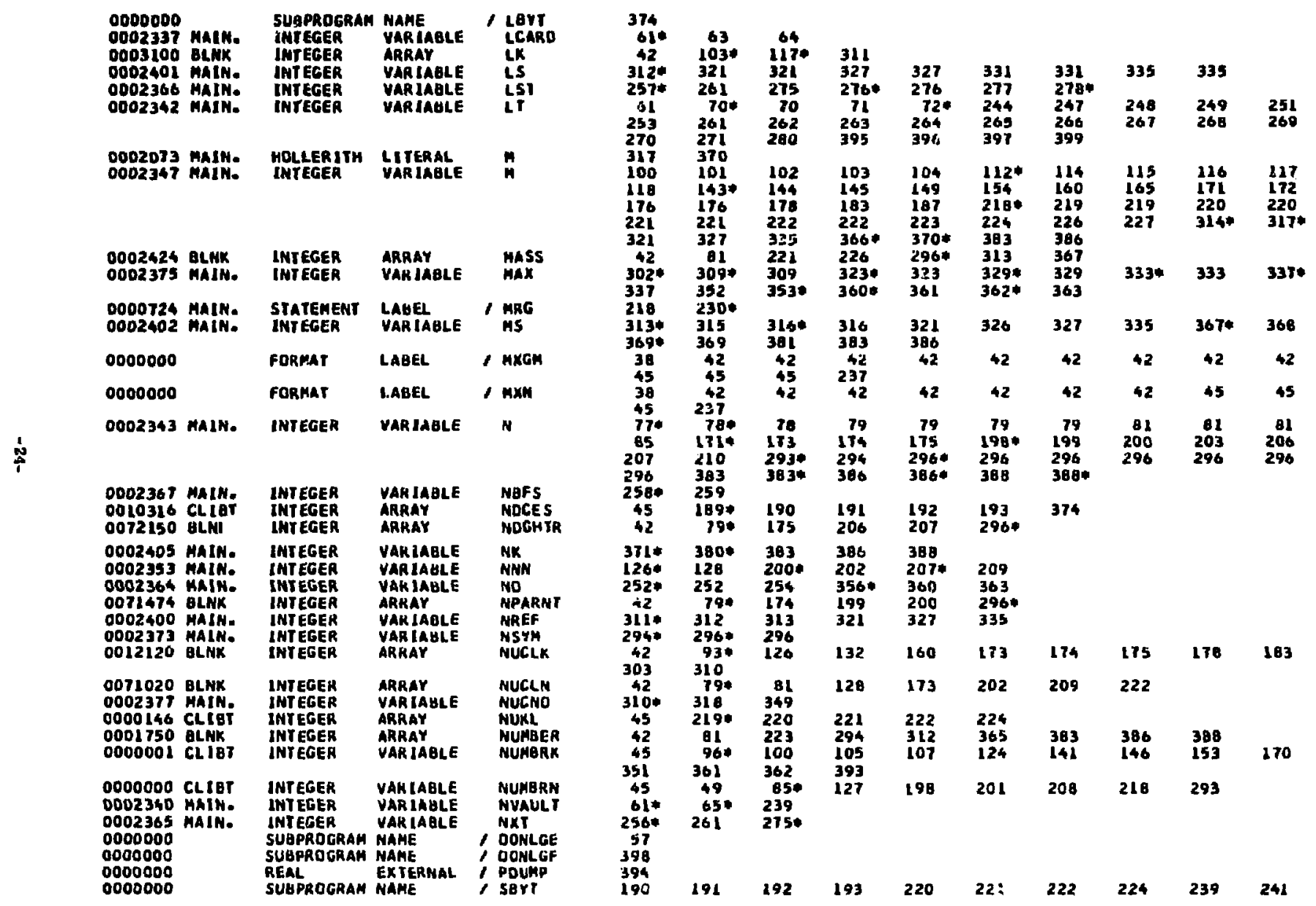




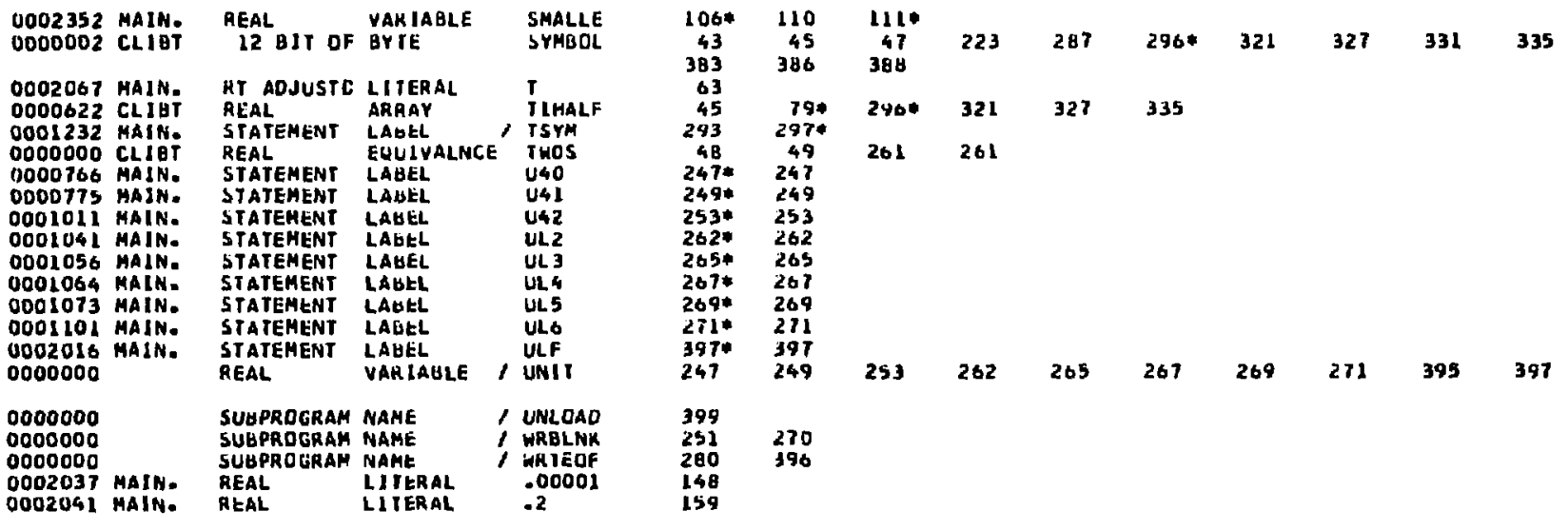




\section{The LINEARITY Program}

This program is used to determine the coefficients of the polynomial that best describes the non-linearity of a counting system. The input data can be either pulservoltages or gamma-ray energies along with the corresponding peak positions in a pulse-height spectrum. The data are fitted by the method of least squares to an equation of the following form:

$$
P_{i}=\sum_{j=1}^{N} a_{j}\left(C L_{i}\right)^{j-1}
$$

where

$$
\begin{aligned}
C L_{i} & =\begin{array}{l}
\text { peak position associated with the pulser-voltage or gamma-ray } \\
\text { energy } P_{i}
\end{array} \\
a_{j} & =\begin{array}{l}
\text { one of a sei of coefficients describing the nonlinearity of the } \\
\text { system }
\end{array}
\end{aligned}
$$

The coefficients that are generated are placed on an appropriate Type $\$$ card (see Vol. (If of this report ${ }^{\circ}$ ).

Some systems may be quite nonlinear in the low-channel region. If the equation gives a poor fit in this region, there is a provision for adding a linear component to the lov-channel data (algebraically) betore fitting a polynomial. The input variables COFDEL and COFCHN describe the $y$-axis (zero energy) and s-axis intercepts of this line. If such an adjustrient is made to the daia, the values of COFDEL and COFC FN used must also be placed on the appropriate Type 4 card for that system.

\footnotetext{
Niday and Gunnink, p. 2 .
} 


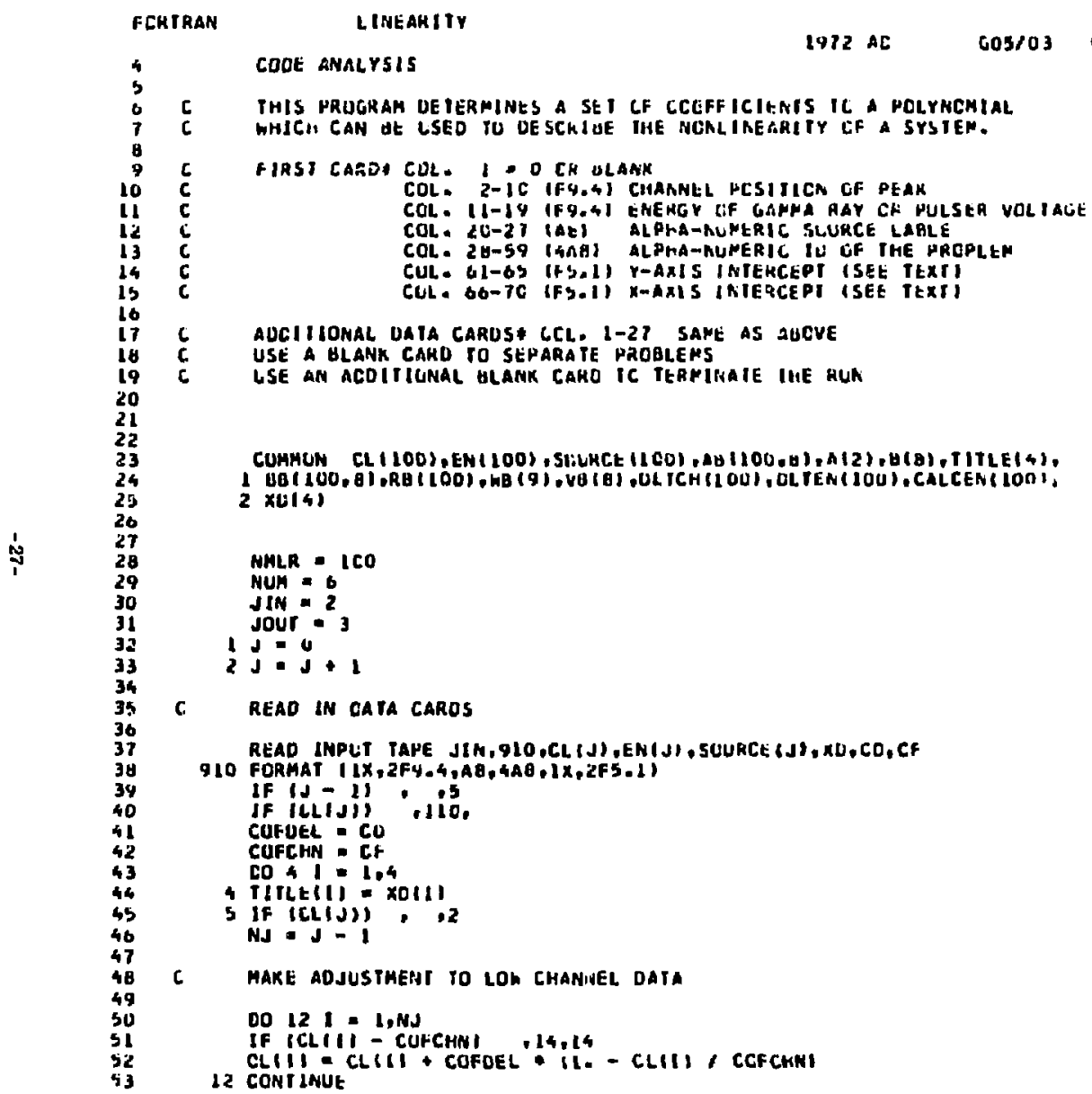

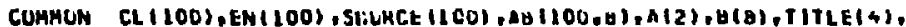

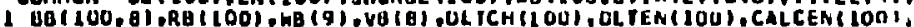
$2 \times(4)$

HALR - ICO

MUN $=6$

JIN $=2$

jour -3

$17=0$

2 J.J+ I

C READ IN cata caros

READ IMPLT TAYE JIM,

910 FORMAT $\mid(X, 2 F 4,4, A B, 4 A B, \mid X, 2 F 5.11$

IF (J-i) if

JFILIJI) MIO

CuFuel - CO

CUFCHN C CF

4 TITEIII:

5 If $(C L(J)), 2$

NJ $=\mathbf{J}-\mathbf{I}$

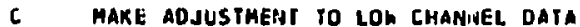

DO $121=1, \mathrm{NJ}$

If ICLIII - CUFCHNi

If ICLIIT-CUFCHNI , 14,14

12 CONIINUE 


\section{c \\ PERFORM LEAST SQUARES FII}

14 DO 100 MRES - 2 , NUM

IB $M$ MRES +2

CO $20 \mathrm{~J}=1, \mathrm{NA}$

$A B(J, 1)=3 . C$

PRO $=$ CL(J)

SPD $=$ PHD

$A B(J, 2)=P R O$

CO $201=3.1 \mathrm{P}$

PRO = PRO

ABIJ, II - PRO

20 cont inve:

CALL MLR (NHLR,NJ, $2, A B, E N, A, B B, R B, W B, Y B$ )

CALL MLA INALR,NJ,IU,AU,EN, I,UA,RU,WE, VUI

\section{co $30 \mathrm{CAL}=$ L.NJ}

CALCEN(J) = $\quad$ (L

35102010

35 CALCEN(J) \& CALCEN(J) + E(I) AB(J, :

DLTCH(J) $=-A B(J, 2)+(L A(J)-A(1)) ; A(2)$

OLTENIJ) = EN(J) - CALCEN(J)

30 CONTINUE

WAJTE OUTPUT IAPE JDUT, $530, N D E G$, IIITLE (J), JEl 4

930 FORHAT IIHL, $/ 1$ 9X.3LHPLLSE ENERGY TC CHANNEL FIT IS II.

i 26HIH ORDEK POLYNOMIAL FOR , HAB)

940 FORHAT IOX,

1 I3HUELTA CHAN =,F4.1,14H*11 CHANAEL/,F4-0,1HI) WRITE OUTPUT TAPE JOUI,950

950 FDRMAT III IIX.3OHCOEFF ICIENTS JF POLYNOMIAL ARE

WRITE DUTPUT TAPE JUUT, $560,(8 / \mathrm{L}), L=2,18)$

960 FORMAT $\mid /$ LSK,E $(4-5)$

WHITE DUTPUT TAPE JUU1,970

970 FORMAT (// IIX,47H CHANAEL

1 , ISHUELTA CHAN. SOURCE $/ 1$

ENERG CALC. ENERGY CELTA EN.

(J),

1 DLTEN(J),OLTCH(J), SQURCE (J), J = I,NJ I

980 FORMAT $(3 X, 13, F 9,3, F 11,3, F 13,3, F 11,3, F 12.3,4 \times, 48)$

200 CONT IINUE

$$
\text { GO TU } 1
$$

110 CALL EXIT

ENC 


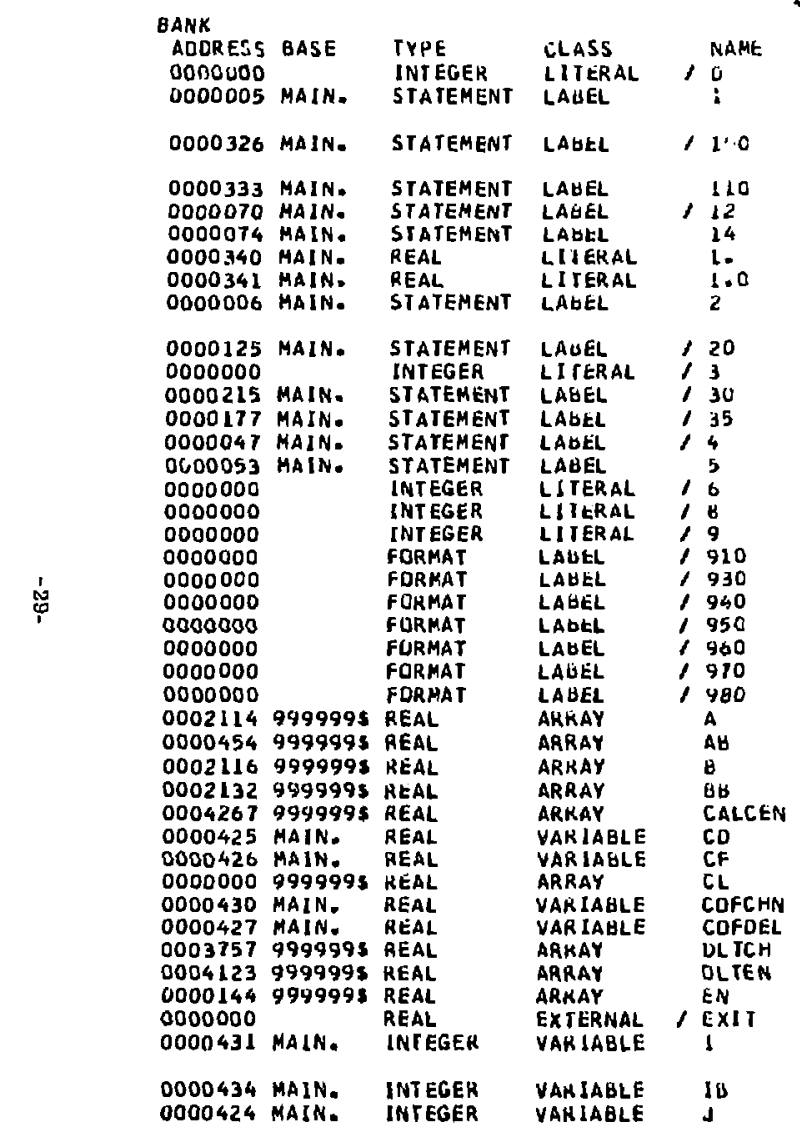

** Code analysis ***

SCURCE LINE REFERENCES

$\begin{array}{ll}32 & \\ 32 * & 39 \\ 14 & 77 \\ 25 & 25 \\ 57 & 574 \\ 40 & 99 \\ 20 & 53 \\ 51 & 51 \\ 52 & \\ 61 & \\ 25 & 30 \\ 17 & \\ 60 & 65 \\ 31 & 65 \\ 73 & 794 \\ 75 & 76 \\ 25 & 25 \\ 39 & 45 \\ 29 & \\ 25 & 25 \\ 25 & \\ 37 & 38 \\ 41 & 834 \\ 94 & 86 \\ 67 & 86 \\ 89 & 90 \\ 91 & 934 \\ 95 & 96 \\ 25 & 70 \\ 25 & 61 \\ 25 & 71 \\ 25 & 70 \\ 25 & 74 \\ 374 & 41 \\ 37 & 42 \\ 25 & 37 \\ 42 * & 51 \\ 41 * & 52 \\ 25 & 770 \\ 25 & 78 \\ 25 & 37 \\ 99 & \\ 43 * & 44 \\ 75 * & 76 \\ 58 * & 59 \\ 32 * & 33 \\ 60 * & 61 \\ 77 & 77 \\ 95 & 95 \\ & \\ 90\end{array}$

I NAME IN ND EXECUTABLE INSTRUCTION C LENOIES ORIGINAL COUNT IN CLICHE

- lenotes mane oefined oR label uSE

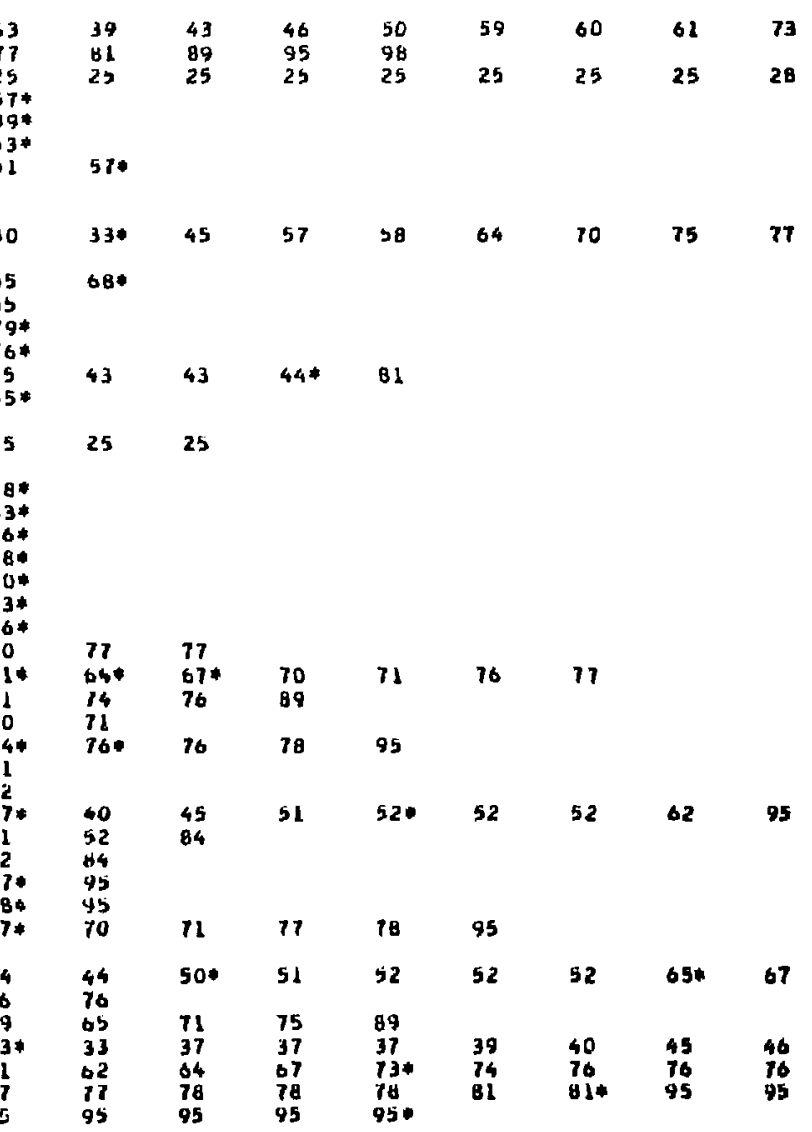




\begin{tabular}{|c|c|c|c|c|c|c|c|c|c|c|}
\hline $\begin{array}{l}0000422 \\
0000423 \\
0000000 \\
0000000 \\
0000435 \\
0000432 \\
0000420\end{array}$ & $\begin{array}{l}\text { MAIN. } \\
\text { MAIN. } \\
\text { MAIN. } \\
\text { MAIN. } \\
\text { MAII }\end{array}$ & $\begin{array}{l}\text { INTEGER } \\
\text { INTEGER } \\
\text { INTEGER } \\
\text { SUBPROGRAM } \\
\text { INTEGER } \\
\text { INTEGER } \\
\text { INTEGER }\end{array}$ & $\begin{array}{l}\text { VAR IABLE } \\
\text { YAK IABLE } \\
\text { VAR IABLE } \\
\text { NAME } \\
\text { VAR IABLE } \\
\text { VAK IABLE } \\
\text { VAR IABLE }\end{array}$ & $\begin{array}{l}\text { JIN } \\
\text { JOUT } \\
L \\
\text { MLR } \\
\text { NOEG } \\
\text { NJ } \\
\text { NMLR }\end{array}$ & $\begin{array}{l}30 * \\
11 \% \\
89 \\
70 \\
59 \% \\
46 * \\
28 *\end{array}$ & $\begin{array}{l}37 \\
61 \\
096 \\
71 \\
41 \\
50 \\
70\end{array}$ & $\begin{array}{l}60 \\
11\end{array}$ & 87 & 71 & 73 \\
\hline $\begin{array}{l}0000433 \\
0000421 \\
0000436 \\
0003572 \\
0000310 \\
0000437 \\
00021116 \\
0003747 \\
0003736 \\
0004433\end{array}$ & $\begin{array}{l}\text { MAIN. } \\
\text { MAIN. } \\
\text { NAIN. } \\
4999995 \\
9999995 \\
\text { MAIN. } \\
9999998 \\
949999 \\
9999998 \\
9999995\end{array}$ & $\begin{array}{l}\text { INTEGER } \\
\text { INIEGER } \\
\text { REAL } \\
\text { REAL } \\
\text { REAL } \\
\text { REAL } \\
\text { REAL } \\
\text { REAL } \\
\text { REAL } \\
\text { REAL }\end{array}$ & $\begin{array}{l}\text { VAK IARLE } \\
\text { VAR IAULE } \\
\text { VAR IABLE } \\
\text { ARMAY } \\
\text { ARKAY } \\
\text { VAR JABLE } \\
\text { ARRAY } \\
\text { ARRAY } \\
\text { ARKAY } \\
\text { ARRAY }\end{array}$ & $\begin{array}{l}\text { NRES } \\
\text { NUH } \\
\text { PRD } \\
\text { RE } \\
\text { SOURCE } \\
\text { SPD } \\
\text { TIILE } \\
\text { VU } \\
\text { WU } \\
\text { XU }\end{array}$ & $\begin{array}{l}57 * \\
29 * \\
62 * \\
25 \\
25 \\
63 * \\
25 \\
25 \\
25 \\
25\end{array}$ & $\begin{array}{l}58 \\
57 \\
63 \\
10 \\
370 \\
66 \\
446 \\
70 \\
70 \\
370\end{array}$ & $\begin{array}{l}64 \\
71 \\
95 \\
81 \\
11 \\
71 \\
44\end{array}$ & 660 & 66 & 67 \\
\hline
\end{tabular}




\section{The XDET Program}

One of the parameters used for geometry calculations in GANANAI is the distance from the detector surface to the window. Although an approximate vaiue of this distance is generally available from assembly specifications, the program XDET is used to experimentally determine or to certify this distance. The input data needed consists of a series of integrated peak counts iaken at different distances from the detector window, the detector radius and height, the gamma-ray energy, and an initial estimate of the window to detector surface distance.

The program proceeds to refine this trial value by seeking a best "least-squares" fit of the data to the foilowing expression: (See Fig. 1)

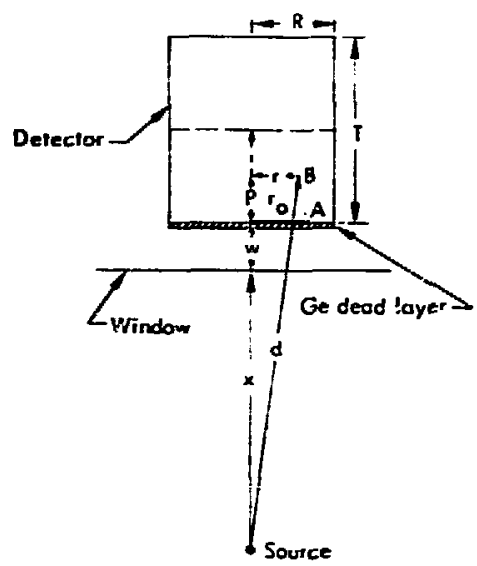

Fig. 1. Geometric relationships for a point source and a cylindrical detector.

$$
\frac{t_{i}}{y_{i}}=c\left[\left(x_{i}+w+p\right)^{2}+r^{2}\right]
$$

where

$y_{i}=$ the peak count in time $t_{i}$

$x_{i}=$ source-to-window distance

$w=$ window-to-detector surface distance

$p=$ effective photon penetration

$r=$ effective radius of interaction

$c=$ proportionality constant

Since the equation is nonlinear, a Taylor's expansion is made about the trial values for $c$ and $(w+p)$. The values of $p$ and $r$ are calculated in the program by using the formulas described in Section III D of Vol. I. ${ }^{2}$ The Newton-Raphson iterative technique is then used to obtain the best least-squares answers for the quantity $(w+p)$. The verious parameters are printed out along with tables showing the precision of the fit.

One must use caution when working with low energy sources. Many detectors have a significant "dead layer" of $\mathrm{Ge}$ in front of the active region. Many of the gamma rays from close-in sources will strike this "dead layer" at angles less than the normal to the surface and they will consequently suffer more attenuation than that experienced

\footnotetext{
${ }^{2}$ R. Gunnink and J.B. Niday, Computerized Quantitative Analysis by Gamma Ray Spectrometry. Vol. I. Description of the GAMANAL Program, Lawrence Livermore Laboratory, Rept. UCRL-51061. Vol. I (1972).
} 
$h$; 7 .mma rays from a source placed at a large distance. The results from XDET for $(w+p)$ arid consequently for $w$ will tend to be too large for such low energy sources unlews some compensation for the above phenomenon is incorporated into the calculatinns. 


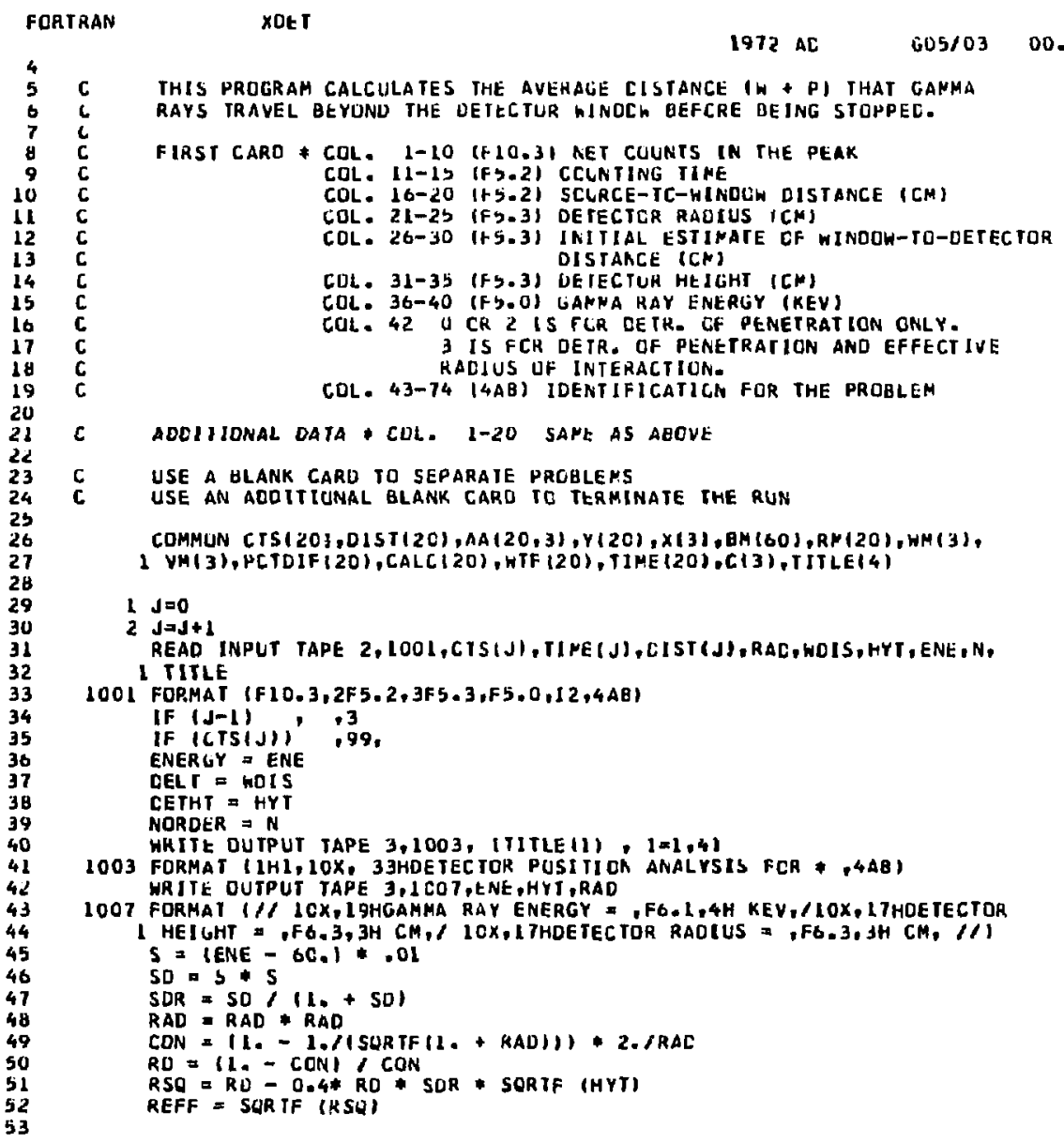


Calculate an efFective absorption coefficient fon ge cetecior

ENE = ENERGY *.001

XMU $=-2.316+4.2$ EXPF $\{-1.434-0.478$ 1CGF (ENE)

XMU $=$ EXPF $\{X M U)$

c Now Calulate efFective gama Ray penetratich INTC IHE detector

C = XHU O OETHT

EXPON = Ext't $(-0)$

$P=11,-10 *$ EXPON, 11. - EXP(N)), XMU

3 IF (CTSIJ)I49, .2

NUM $J-1$

IF (NORDER)

NOROEK $=2$
CD $5 \mathrm{~N} 2=1$, NUM

4 CD $5 J=1, N U M$

HTF(J) $=1.0, \operatorname{SQRIF}(C I S(J))$

5 CUNTINUE

G UISTSNUMI + DELT + P

$B \Rightarrow(\theta+8)+R S O$

CONSI $=$ CTSINUMI 0, TIME INUN)

LCNI - 0

MRTE DUTPUT TAPE 3.1002

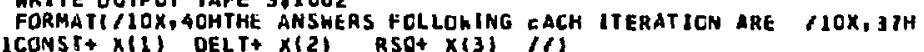

HRITE DUTPUT TAYE $3,1 C 05, C O N S T$ DELT ORSU

10 DO $20 \mathrm{~J}=1 . \mathrm{NHAM}$

DEUISTIJ) + DELT

$A X=1.0)(10 * D)+R S U)$

AA $(J, i)=A X+H T(j)$

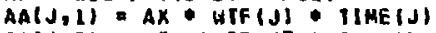

$A A(J, 2)=-2$. CONST $D * A X * A A(J, 2$

IF INURDER - 2120.20 .

$A A(J, 3)=-C O N S T$ * AX *AAIN, 1

20 Y(J) : (CTSIJ) - CUNST TIME(J) AX) - XJFIJ)

LCNT = LCNI \& I

DELT = OELT $+\times(2)$

CUNS I CONST + COL11

IFINURDER -2) 22,22,

ASO $=R S O+X(3)$

22 WRITE DLTPLT TAPE 3,1CO5, LONST, DELT, RSE

1 COS FORMAT I BX, JEL 3.4$\}$

IFILCNT - 20 ) :.24

If (AUSF $(x(2)) \div .003), 10,10$

24 SUMR $=0.0$

COF = NUM - NOROER

DO $30 \mathrm{~T}=\mathrm{I}, \mathrm{NUH}$ 


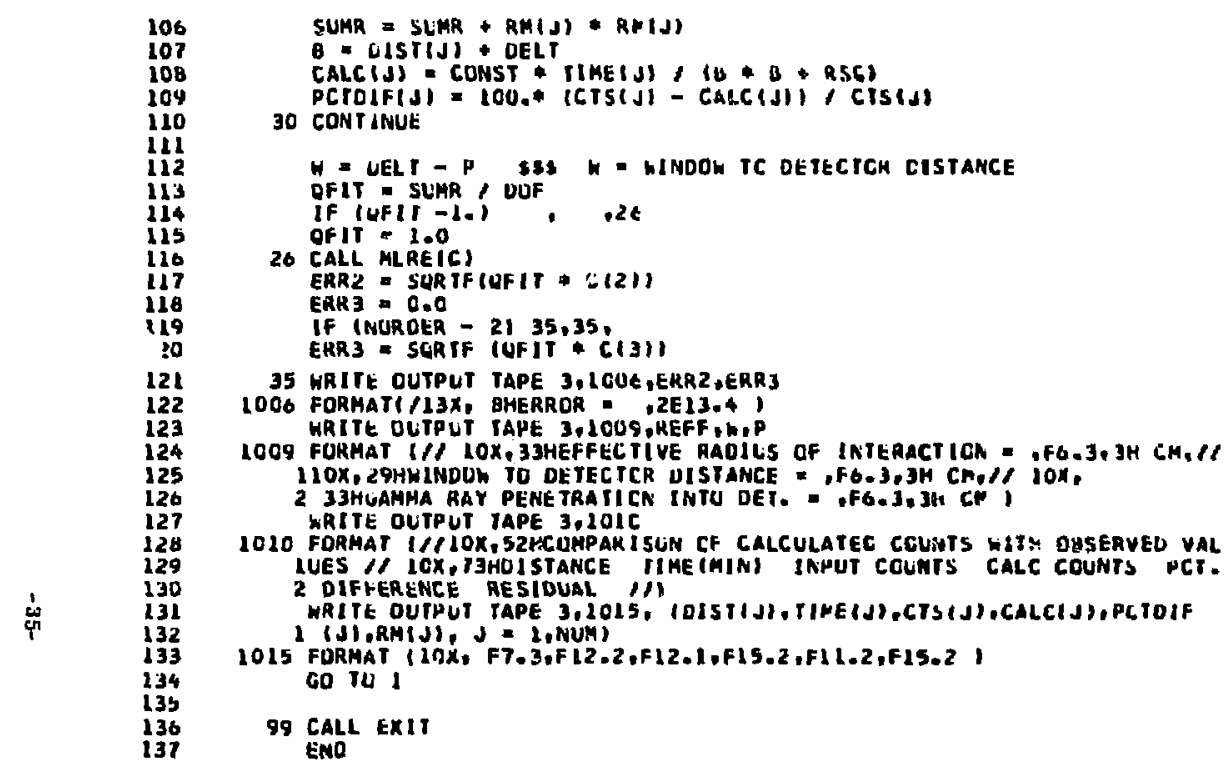

